\title{
Spatially Resolved Localized Vibrational Mode Spectroscopy of Carbon in Liquid Encapsulated Czochralski Grown Gallium Arsenide Wafers
}

\section{Waifan Yau}

\author{
M.S. Thesis
}

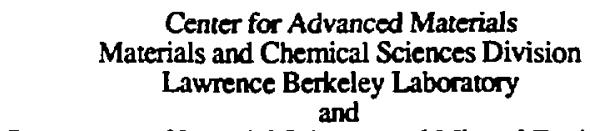

Department of Material Sciences and Mineral Engineering University of California, Berkeley

April 1988

This work was supported by the Director, Office of Energy Research, Office of Basic Energy Sciences, Materials Science Division of the U.S. Department of Energy under Contract No. DE-AC03-76SF00098. 


\section{ABSTRACT}

Subsitutional carbon on an arsenic lattice site is the shallowest and one of the most dominant acceptor in semi-insulating Liquid Encapsulated Czochralski (LEC) GaAs. However, the role of this acceptor in determining the well known ' $W$ ' shape spatial variation of neutral EL2 concentration along the diameter of a LEC wafer(Ref.7) is not known. In this thesis, we attempt to clarify the issue of the carbon acceptor's effect on this ' $W$ ' shaped variation by measuring spatial profiles of this acceptor along the radius of three different asgrown LEC GaAs wafers. With localized vibrational mode absorption spectroscopy, we find that the profile of the carbon acceptor is relatively constant along the radius of each wafer. Average values of concentration are 8 $\times 10 \mathrm{E} 15 \mathrm{~cm}^{-3}, 1.1 \times 10 \mathrm{E} 15 \mathrm{~cm}^{-3}$, and $2.2 \times 10 \mathrm{E} 15 \mathrm{~cm}^{-3}$ respectively. In addition, these carbon acceptor LVM measurements indicate that a residual donor with concentration comparable to carbon exists in these wafers and it is a good candidate for the observed neutral EL2 concentration variation. 


\section{ACKNOWLEDOMENTS}

I would like to thank my research advisor, Professor Eugene Haller for his guidance and support throughout this work. Dr. Waldek Walukiewicz is gratefu!ly acknowledged for his helpful advice and ongoing interest in this work.

Special thanks to Dr. Robert McMurray for his technical advice and many helpful discussions. I would like to express my appreciation to Jeffery Beeman for his technical assistance. I would also like to thank Dr. Kin Man Yu and Dr. Robert McMurray for commenting on the manuscript. David Bliss of MACom. is acknowledged for supplying the GaAs samples.

I also like to acknowledge Professor Eicke Weber of the Material Science and Mineral Engineering Department and Professor John Smith of the Electrical Engineering and Computer Science Department for reviewing and making many valuable comments on this thesis.

This work was supported by the Director, Office of Energy Research, Office of Basic Energy Sciences, Materials Science Division of the U. S. Department of Energy under contract DE-AC03-76SF00098. 
TARLE OF CONTENTS

Page

1 Introduction.............................................................................. 1

1.1 Technological Importance and Properties of GaAs....... 1

1.2 Semi-insulating (SI) GaAs................................................. 3

1.2.1 EL2 in SI GaAs............................................................. 5

1.2.2 Carbon in SI GaAs.................................................... 6

1.3 Spatial Profile of Carbon in GaAs Measured by

Localized Vibrational Mode Spectroscopy......................... 8

2: Instrumentation........................................................................... 12

2.1 Theory of Fourier Transform Spectroscopy..................... 12

2.1.1 Advantage of an interferometer over a grating spectrometer................................................... 16

2.2 Digilab FTS-20E Interferomter............................................ 17

2.3 Air Products LT-3-110 Continous Flow Crystat............... 22

2.4 Operation of the Interferometer plus Cryostat for

Measuring Spatially Resolved Carbon LVM................... 25

$3 \quad$ Results............................................................................................ 31

3.1 Linear Absorption Spectroscopy....................................... 31

3.1.1 Linear Absorption Spectroscopy of Carbon's

LVM in GaAs............................................................ 34

3.2 Charge State Sensitive LVM lines of Carbon in GaAs... 37

4 Discussion.............................................................................................. 44

4.1 Two Inch Diameter LEC GaAs Wafer............................... 44

4.1.1 The 'Two Defect' Model for SI GaAs..................... 44 
4.1.2 The 'Three Defect' Model for SI GaAs.................. 46

4.2 The $4 \mathrm{~mm}$ Thick Three Inch Diameter LEC GaAs

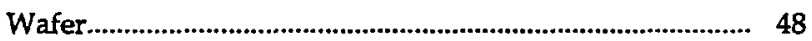

4.2.1 The 'Two Defect' Model for SI GaAs..................... 48

4.2.2 The 'Three Defect' Model for SI GaAs.................. 49

4.2.3 The 'Four Defect' Model for SI GaAs.................... 49

4.3 The $2 \mathrm{~mm}$ Thick Three Inch Diameter LEC GaAs

Wafer.

4.3.1 'Two Defect' and 'Three Defect' models for SI GaAs........................................................... 50

4.3.2 The 'Four Defect' Model for SI GaAs....................... 51

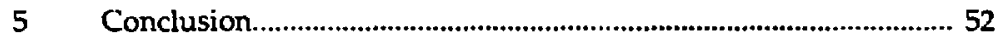

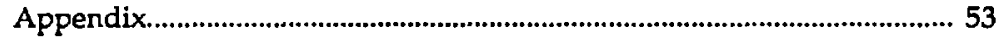

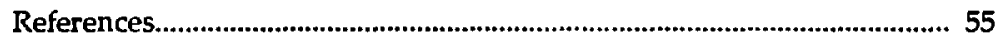




\section{GALLIUM ARSENIDE}

Gallium arsenide (GaAs) has been widely accepted as the "next generation semiconductor material". GaAs will not replace silicon (Si) in the future, rather, it is a promising material for applications where Si fails to provide satisfactory performance (for example, optoelectronic devices, high speed ( $>10$ $\mathrm{GHz}$ ) switching field effect transistors, and microwave generators). The superiority of GaAs is mainly due to its particular band structure. GaAs is a direct band-gap semiconductor in which the minimum of the conduction band (CB) lies directly above the maximum of the valence band (VB), both of which are located at the center of the Brioullion zone $(k=0)$ in reciprocal lattice space (Fig.1.1). Electronic transition processes across the band gap (1.45eV in magnitude at room temperature) are highly efficient since no phonon assistance is required. For this reason, $\mathrm{GaAs}$ is an excellent material for fabricating various optoelectronic devices and integrated circuits. Another interesting feature of the GaAs band structure is large curvature $\left(d^{2} E / d k^{2}\right)$ at the minimum of its conduction band. The effective mass of electrons occupying the minimum of the CB is inversely proportional to the curvature of the CB (Ref. 1). In the case of GaAs, this large curvature (Fig. 1.2) leads to an effective mass value of $0.072 x$ free electron mass (Ref. 2), which is very small compared to that of silicon (0.97 $x$ free electron mass (Ref. 2)). This difference accounts for almost an order of magnitude increase in low electric field electron mobility compared to Si. As a result, GaAs field effect transistors can operate at frequencies as high as 20 $\mathrm{GHz}$ (Ref.19). Thus, GaAs is a more suitable material for very high speed components. Besides the larger curvature near the minimum of the $C B$, a local 


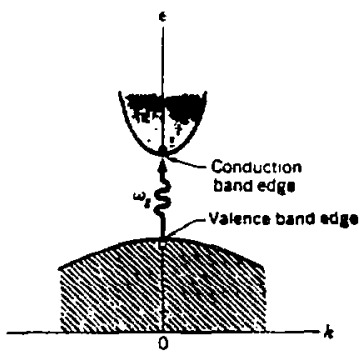

\{al

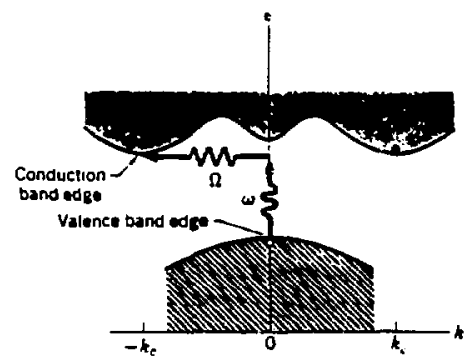

(b)

XBL 885-1828

Fig.1.1

(a) Band diagram of a direct band gap semiconductor like GaAs.

(b) Band diagram of an indirect band gap semiconductor like Si

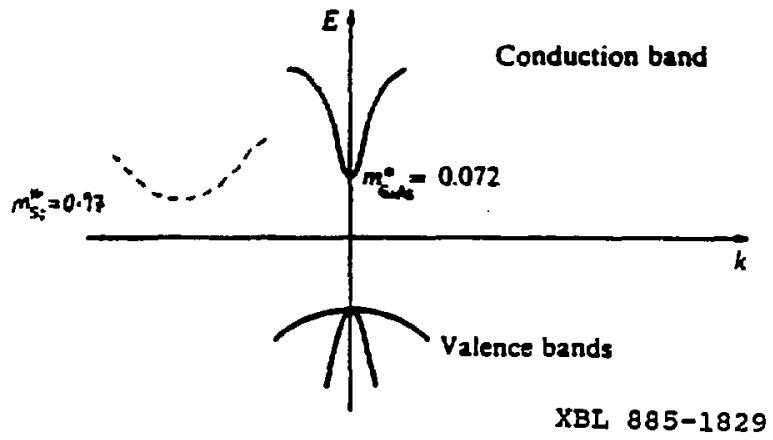

Fig. 1.2 The curvature of the minimum of the CB of GaAs is higher than that of Si; solid line represents the CB of GaAs and dotted line represents Si. 
minimum at zone boundary "L6" (Ref. 3) along the $<111>$ direction, known as the "satellite band" is also an important feature of the GaAs band structure (Fig.1.3). When the applied electric field exceeds $3300 \mathrm{~V} / \mathrm{cm}$ (Ref.1), electrons from the minimum of the CB will spill over into this satellite minimum. Microwave emission is made possible since devices operating at this condition will have negative differential impedance. The well known Gunn effect (Ref. 20) is making use of this phenomenon and GaAs Gunn oscillators have many applications in the microwave industry.

Gallium arsenide is a compound semiconductor with a crystal structure resembling that of the zinc-blende structure (Fig.1.4). The two- atom basis introduces extra complications in terms of crystal imperfections since stoichimetery as well as impurities play major roles in determining crystal quality. Typical melt grown (Czochralski) GaAs crystals may contain as many as 10E5 $\mathrm{cm}^{-2}$ dislocations, $10 \mathrm{E} 16 \mathrm{~cm}^{-3}$ native defects, and $10 \mathrm{E} 15 \mathrm{~cm}^{-3}$ impurities. It is not hard to understand that the performance of GaAs devices depends significantly on the balance of each type of residual defect present, such as carbon, EL2, iron, arsenic antisite, and silicon.

\subsection{SEMI-INSULATING(SI) GALLIUM ARSENIDE}

One of the main objectives of GaAs crystal growth is to grow semi-insulating crystals for integrated circuits applications. That is, to grow crystals with the Fermi level close to the middle of the band gap. Resistivity of such crystals can be as high as $10 \mathrm{E} 8 \mathrm{ohm}-\mathrm{cm}$. The advantage of using semi-insulating crystals is straight forward: integrated circuits fabricated on this material do not require electrical isolation regions (field exide or reverse biased junction) to seperate one device from another or from the substrate. The absence of parasitic isolation junctions or oxide capacitances leads to higher speed performarıce. 


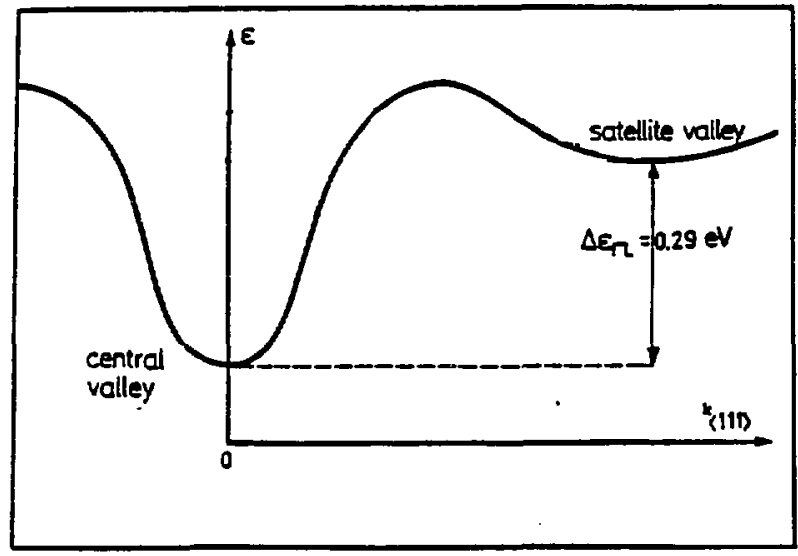

XBL $885-1830$

Fig. 1.3

Satellite valley L6 of GaAs is located $0.290 \mathrm{~V}$ above the central minimum of the CB along the <111> direction at zone boundary.

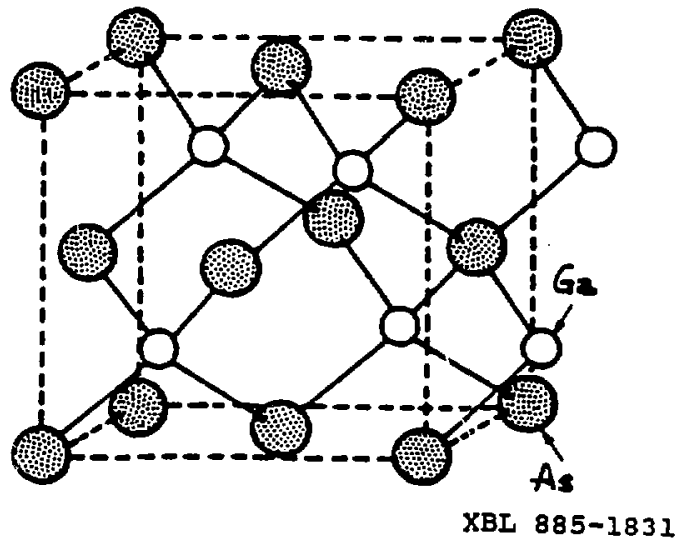

Fig. 1.4

Zinc blende structure of GaAs: the larger spheres represent arsenic atoms and the smaller spheres represent gallium atoms; lattice constant is 5.65 angstrom. 
Also, more devices can be fabricated within the same area of a substrate reducing the cost per device and increasing the high speed performance.

\subsubsection{EL2 in semi-insulating Gallium Arsenide}

The semi-insulating condition can be achieved by exact compensation between donors and acceptors (a theoretical case), purification ( with impurity concentration less than $10 \mathrm{E} 6 \mathrm{~cm}^{-3}$; this is another theoretical case), or by introducing a dominant mid-gap defect or impurity into the crystal. The midgap defect used in today's technology is called EL2. Although the exact identity of EL2 is not fully understood, many of its properties have been thoroughly studied. EL2 is a mid-gap double donor with "photo-quenching" (Ref. 6) behavior (Fig. 1.5). In brief, the "photo-quenching" process involves absorption of $1.1 \mathrm{eV}$ photons by neutral EL2 centers. The photon absorption leads to a change of electronic configuration of EL2 and excites the normal EL2 donor into its metastable state EL2*. EL2* is not electrically active. At temperatures above -i40K, EL2* will decay back to normal EL2 ; but at temperatures below 140K, EL2" will be stable. After all EL2 donors are transformed into EL2*, the material is "photo-quenched" and the Fermi level will have moved towards the valence band.

EL2, togeitier with residual shallow acceptors $(A)$ and residual shallow donors $\left(D_{S}\right)$, determine the position of the Fermi level. In order to demonstrate this point, consider two hypothetical situations shown in figures 1.6 and 1.7 . In figure 1.6, the conditions $[A]>\left[D_{S}\right]$ and $[E L 2]+\left[D_{S}\right]>[A]$ lead to a Fermi level position near mid-gap, yielding a semi-insulating crystal. In figure 1.7, the condition $[A]>[E L 2]+\left[D_{S}\right]$ leads to a Fermi level position near the acceptor level, which yields a p-type crystal. The above illustration shows that the relative concentrations of EL2, shallow acceptors, and shallow donors determine the position of the Fermi level. 


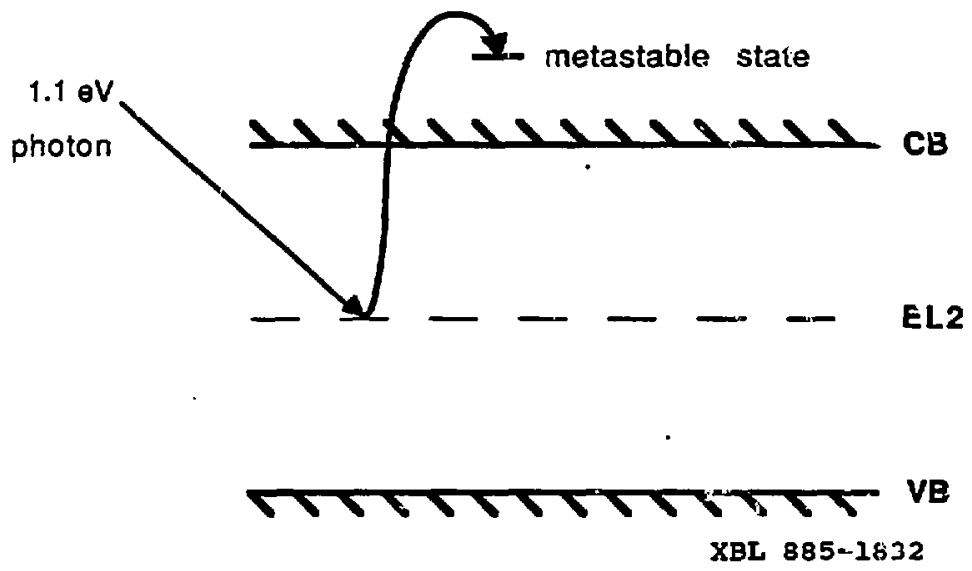

Fig. 1.5 Schematic demonstrating "photo quenchirg" of EL2; the metastablu stata does not belong to the energy band and does not participate in any electical activilies.

\subsubsection{Carbon in semi-insulating Gallium Arsenlde}

Subsitutional carbon an an arsenic site in GaAs was first cietecteci by Newman in 1971 (Ref. 5). Being a group IV element, carbon can behave amphoterically just as subsitutional silicon (Ref.17) in GaAs. If carbor occupies a gallium site $\left(\mathrm{C}_{\mathrm{Ga}}\right)$, it will act as a donor whereas if ii sits on an arsenic site $\left(C_{A s}\right)$, it will behave as an acceptor. When discussing carbon in GaAs, one always refers to the acceptor state because the conor state has never been identified experimentally. The cartún acceptor has two infrared active signatures: one involves electronic transitions of a bound hole irom its ground state to various bound excited states and to the valence band (Ref.2); the other involves the excitation of the localized vibrational modes (Ref.5). 


\section{SI GaAs}

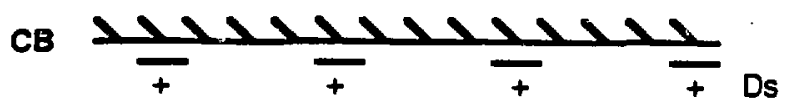

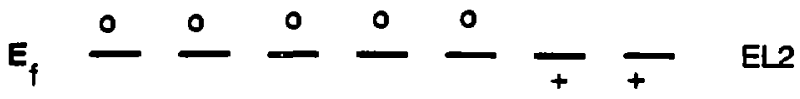

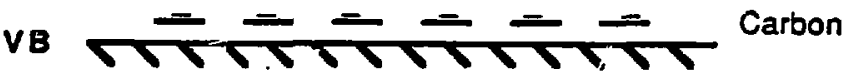

XBI, $885-1833$

Fig. 1.6

Hypothelical defeut model of SI GaAs; the conditions $\left[E[2]+\left[D_{5}\right]>[A]\right.$ and $[A]>\left[D_{S}\right]$ pin the Fermi lovel near the center of the band gaf.

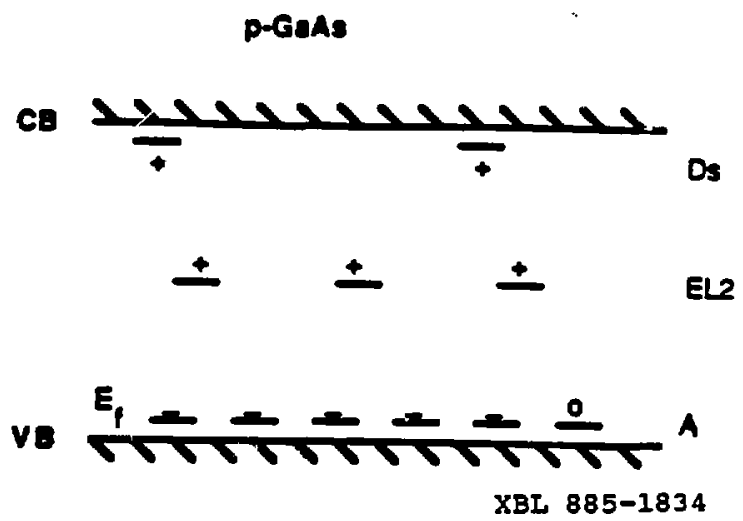

Fig. 1.7 Hypolnetical model of p-type GaAs; the condition $[A]>[E L 2]+\left[D_{S}\right]$ pins the Fermi level near the acceptor energy level. 
Carbon in melt grown GaAs is the shallowest and one of the most dominant residual acceptor. The ground state of the carbon acceptor is located $27 \mathrm{meV}$ (Ref. 4) above the maximum of the VB and its concentration in as- grown melt grown crystals is of the order of $10 \mathrm{E} 15$ to $10 \mathrm{E} 16 \mathrm{~cm}^{-3}$. Because of this high concentration, the carbon acceptor is important in determining the position of the Fermi level. Besides determining the position of the Fermi level as illustrated in figures 1.6 and 1.7, another technologically important aspect of carbon in GaAs is its effect on donor implantation profiles. Because carbon is the shallowest acceptor in GaAs, carbon acceptors are always ionized in semiinsulating GaAs ([EL2] + [DS] > [carbon]). The concentration of implanted donors $\left(D_{i}\right)$ can turn the local region of the crystal n-type only if $\left[D_{i}\right]+\left[D_{s}\right]>$ [carbon]. The amount of electrically active shaliow donors is therefore equal to $\left[\bar{L}_{i}\right]+\left[D_{S}\right]$ - [carbon] (Fig. 1.8) after compensation because the Fermi level will be located close to the conduction band and EL2 will be neutral. (i.e. it does not compensate the acceptor any longer!) Consequently, the donor implantation profile (electrical profile, not physical profile) will be truncated at locations where $\left[D_{i}\right]+\left[D_{s}\right]<$ [carbon] (Fig. 1.9). Spatial variation of carbon concentration in a wafer leads to variation in device performance because device parameters such as junction depth and channel depth will vary in accordance with carbon concentration.

\section{3 SPATIAL PRofile of carbon IN GAas meAsured BY LOCALIZED VIBRATIONAL MODE (LVM) SPECTROSCOPY}

LVM spectroscopy is a very powertul 120 for studying defects in electronic materials (Ret.17). LVMs arise when impurity atoms with mass smaller than that of the host atoms are introduced into subsitutional sites of the 
Implanted donors (Di) compensation

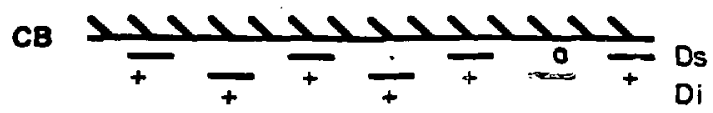

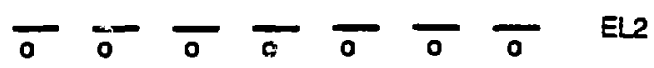

VB

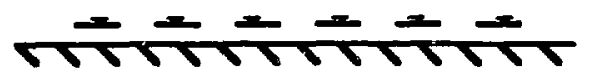

Carbon

XBL 885-1935

Fig. 1.8 Impurity level diagram of a hypothetical SI GaAs crystal with 3 major defects: EL2. $D_{S}$, and carton; when implanted by donors $D_{j}$, only $\left[D_{S}\right]+\left[D_{i}\right]$ - [carbon] shallow donors are elctrically active.

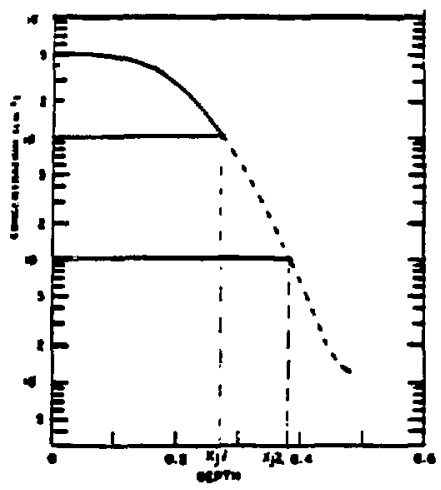

XBL 885-1836

Fig. 1.9

Hypothetical implantation profile of Di in fig. 1.8, truncation of electrical profile depends on background carbon concentration; $x_{j 1}$ represents the junction depth of a higher background [carbon] and $x_{j 2}$ represents the junction depth of a lower [carbon]. 
crystal (Ref.17). LVMs are not travelling waves, instead, they are vibrational modes centered on the defect with their amplitudes of vibration exponentially decreasing with distance away from the defect. The term "localized" refers to the localization of vibration amplituc'e around the defect in real space. Typically, the frequencies of such vibrations lie well above the maximum of the phonon frequency bands, and we observe localization of vibrational states in the frequency space. When the modes are infrared active, they can be observed as infrared absorption lines. Integrated area under each LVM absorption line is proportional to the concentration of the defect to first order and independent of its electronic charge state. For carbon in GaAs, all LVMs are located near 582.4 $\mathrm{cm}^{-1}$ (Ref. 8). Recent studies (Ref. 9) showed that high resolution $\left(0.06 \mathrm{~cm}^{-1}\right.$ ) LVM spectroscopy of this set of lines can also be used to determine the charge state of the carbon acceptor by observing the change in line shape of this set of lines. LVM absorption spectroscopy is thus a very useful tool for studying carbon in GaAs for it provides information regarding absolute concentration as well as charge state.

To understand the frequently exhibited $W$ shaped spatial variation of neutral EL2 concentration along the diameter of each SI GaAs wafers (Ref.7), a detailed study of the spatial distribution of each major defect or impurity is needed in principal to understand this behavior. Carbon has been suggested (Ref.21) to play a major role in this peculiar ' $W$ ' shape. Therefore, as already pointed out in the abstract, we have focused on measuring the spatial distribution of this impurity. With ten or more data points (LVM absorption) along the radius of a wafer, one can interpolate the shape of the carbon profile with sufficient accuracy because the concentration varies relatively slowly. In addition, one can often estimate the profile of the residual shallow donor from these high resolution LVM absorption data points of carbon with EL2 removed 
by photo-quenching (this procedure will be explained in detail in chapters 2,3 , and 4). Thus, carbon spatial maps provide two pieces of information toward the determination of the variation of neutral EL2 concentration. 
In this chapter, both instrumentation and experimental conditions will be discussed. First, an introduction to the theory of Fourier transform spectroscopy will be given. Then, the basic set up of the Digilab FTS-20E vacuum type rapid scan Fourier transform interferometer will be described. Next, the operation of the continous flow helium cryostat (Air Products LT-3-110) will be explained. Finally, the actual experimental procedures will be described.

2.1

\section{THEORY OF FOURIER TRANSFOHM SPECTROSCOPY}

In order to record spatially resolved LVM absorption lines of carbon in GaAs efficiently, a Fourier transform interferometer is used. The reasons for using an interferometer will be explained later in this section. For now, a brief introduction to the theory of operation of an interferometer will be given to familiarize the reader with the subject. First, consider figure 2.1. $S$ is a point source (in reality, $S$ is an extended source and its effect is discussed in section 2.2); depending on the spectral region of interest, it can be a mercury arc lamp for the far-infrared $\left(10-400 \mathrm{~cm}^{-1}\right)$, a glower for the mid-infrared $\left(400-5000 \mathrm{~cm}^{-1}\right)$, and a variety of lamps for the near infrared and the visible $\left(5000-30000 \mathrm{~cm}^{-1}\right)$. Light emitted from the source is collected by an off-axis paraboloidal shaped reflector (P)with its focus at the position of the source. P converts the diverging beam into a parallel beam. This beam then passes through an amplitude splitter or beamsplitter, which is inclined $\mathbf{4 5 0}$ from the initially parallel beam. This parallel beam lo is split into two perpendicular beams: one transmits through the beamsplitter (To) and the other reflects off its front surface (Ro). The reflected beam Ro hits the fixed mirror and bounces back to the front surface of the beamsplitter at $45^{\circ}$ inclination angle since the beamsplitter bisects the mutually perpendicular fixed and moving mirrors. Ro now splits into a reflected 


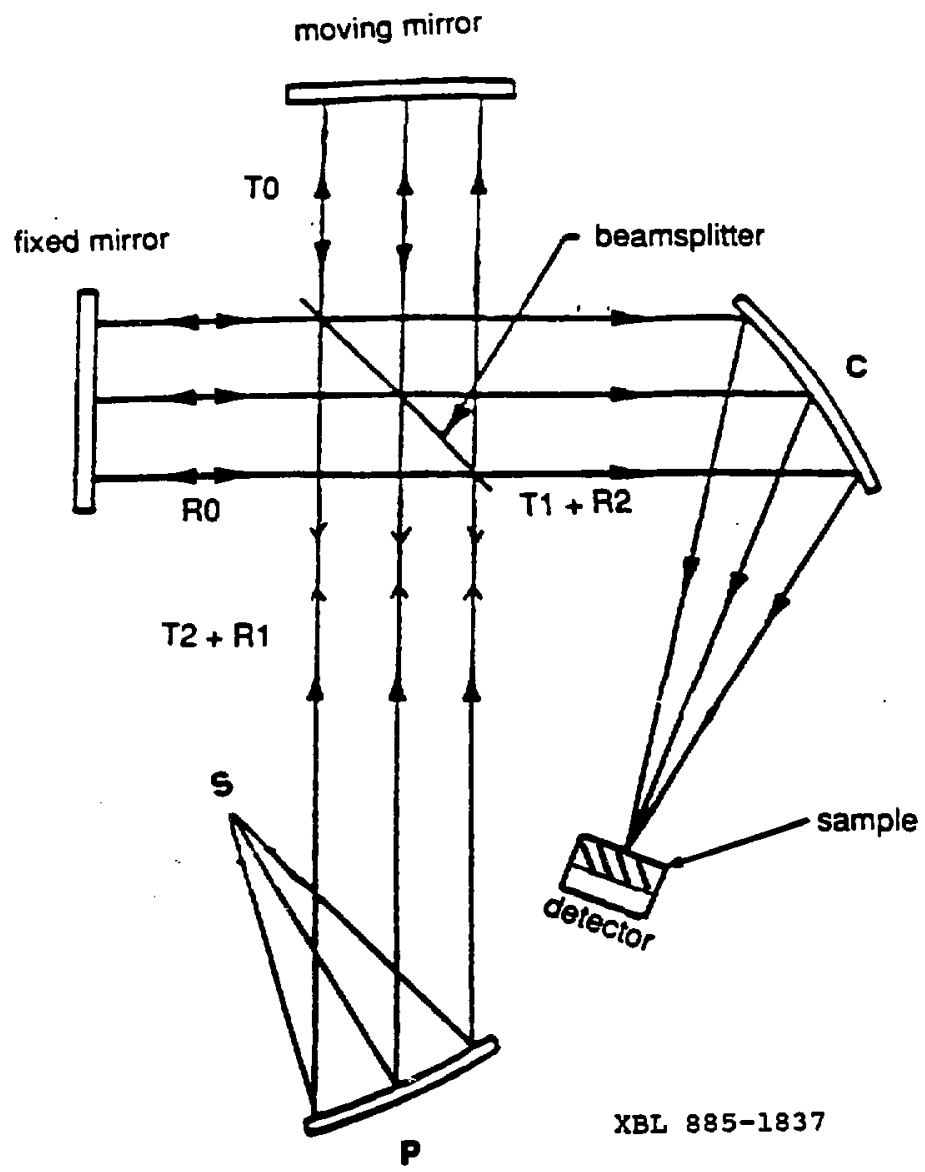

Fig. 2.1

Schematic of an interferometer 
beam $R 1$ and a transmitted beam $\mathbf{T} 1 . T 1$ transmits through the beamsplitter and continues onto the sample whereas R1 returns to the source. Meanwhile, the initially transmitted beam To hits the moving mirror and returns to the back surface of the beamsplitter at $45^{\circ}$ inclination angle. To also splits into a reflected beam $R 2$ and a transmitted beam T2. T1 and R2 form a net transmitted beam heading toward the sample while R1 and T2 retum to the source. This net transmitted beam is focused by the converging reflector $C$ onto the sample. As the moving mirror scans, the detector will receive a characteristic interference pattern of the source.

For a white light source, each photon wavelength of the source will produce its own characteristic interference pattern as a function of optical path difference and the intensity of the source at that particular band at the detector. Every wavelegth band will produce a cosine variation in intensity as a function of optical path difference between R2 and T1 as shown in equation 2.1:

$$
l(x, w)=0.5 l(0, w)(1+\cos (2 \pi w x))
$$

The term $I(0, w)$ stands for the intensity received by the detector at zero optical path difference of the wavenumber band centered at ' $w$ '. ' $x$ ' is the optical path difference between the two recombined beams. $I(x, w)$ is the intensity contribution of this particular band recieved by the detector as a function of optical path difference. When considering all frequencies, the recorded signal as a function of optical path difference is a linear superposition of each corresponding wavenumber band as shown in figure 2.2. The intensity as a function of any finite path difference minus half the intensity of all frequencies at zero path difference is called the interferogram of the source. It is given by equation 2.2: 


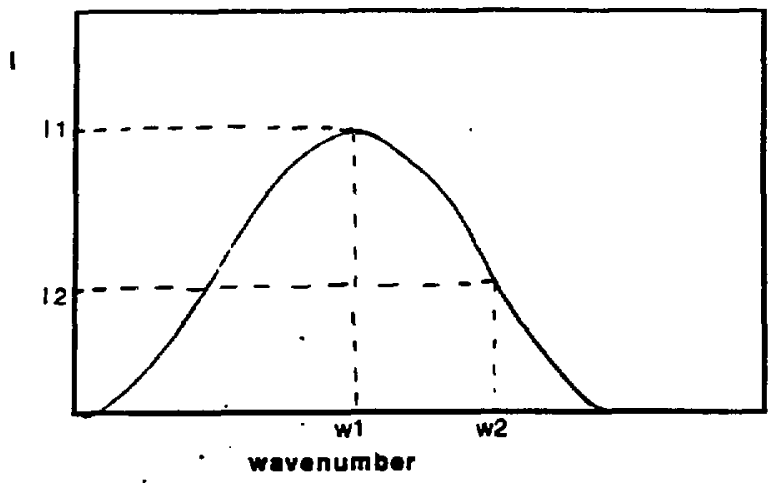

(a)

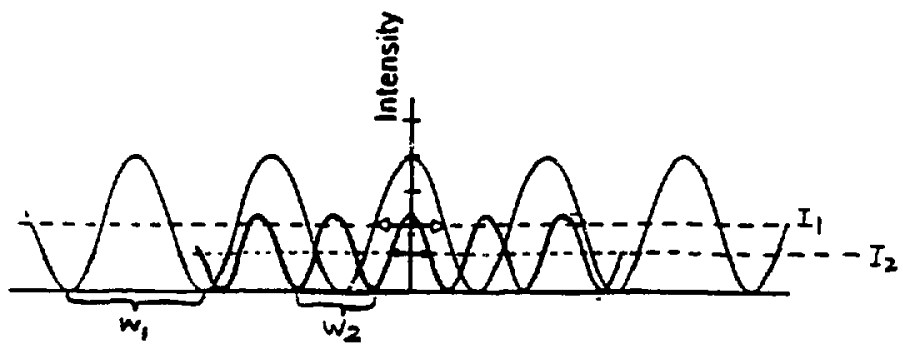

(b)

XBL $885-1838$

Fig. 2.2 (a) Intensity spectrum of an arbitrary source.

(b) Contribution to the interferogram from two frequencies: w1 and w2. 


$$
\begin{aligned}
& I(x)^{*}-0.5 I(0)^{*}=\int I(x, w) \cos (2 \pi w x) d w \\
& I(x)^{*}=\int I(x, w) d w ; I(0)=\int I(0, w) d w
\end{aligned}
$$

By Fourier analysis (Ref. 10), the intensity spectrum of the source can be shown as the inverse Fourier cosine transform of the interferogram as demonstrated by equation 2.3 (equation 2.3 is derived in the appendix).

$$
B(w)=\int(1 / \pi)[l(x)-0.5 l(0)] \cos (2 \pi w x) d x
$$

\subsubsection{Advantages of an interferometer over a grating spectrometer}

Grating spectrometers and interferometers are both used for IR spectroscopy. However, the latter has two important advantages over the former which allow significant reduction in data collection tiı.7e for identical signal to noise ratio and resolution in a specific spectral region. The first of the two is the Jaquinot (Ref. 11) or throughput advantage. When comparing the same resolving power (w/dw), the solid angle of the entrance slit subiending on the collimator mirror of a grating spectrometer depends on the ratio of the length of the entrance slit to the focal length of the collimator mirror (this ratio is typically 0.03). For an interferometer, this restriction does not apply because the solid angle subtended by the source is fixed by its resolving power. Thus, when a comparsion is made between the throughput of a grating spectrometer and an interferometer with identical sources, resolving power, and collimator mirror, one finds that approximately 200 times more power can pass through an interferometer. Assuming noise which is independent of intensity (which is usually the case for mid-infrared detectors), an interferometer can collect data 200 times faster for a given signal to noise level. 
The second advantage of an interferometer is the Felleget (Ref. 12) or multiplex advantage. This advantage arises from the fact that the detector of an interferometer sees all wavelengths at the same time while the detector of $a$. grating spectrometer only sees photons coming from a narrow wavelength band. The scanning time of a grating spectrometer with a specific signal to noise ratio is $M \times T$ where $M$ is the number of spectral subintervals (typically 1OE4) and $T$ is the time spent in collecting data for each subinterval.The signal to noise ratio of each subinterval is proportional to T1/2. For an interferometer with the same total scanning time M.XT, the signal to noise ratio of the spectrum is proportional to $\left(M \times T^{1 / 2}\right.$. For identical experimental conditions with detector noise independent of intensity, one can show that the signal to noise ratio (S/N) of an interferometer is $M^{1 / 2}$ times better than the $S / N$ of a grating spectrometer.

\subsection{DIGILAB FTS-20E INTERFEROMETER}

All data regarding carbon in GaAs in this work are collected by a rapid scan type Fourier transform interforomoter (Digilab FTS-20E). The entire interferometer is enclosed in vacuum so that absorption due to carbon dioxide and water vapor is minimal. The interforomoter is divided into four chambers as shown in figure 2.3. The moving mirror assembly and the light source are located in chamber one. The interferometer is set up for mid infrared operation with a resistively haded coramic glowbar as its radiation source. The glowbar is apertured to an area of $10 \mathrm{~mm} \times 5 \mathrm{~mm}$. The finite size of the IR source sets a fundamental limit to the resolving power of the interforometer because light rays which originate from different parts of the source reflected off the paraboloid mirror are not perfectly parallel. Consequently, the upper limit of resolving power sot by this effect is approximately 3000 as given by equation 2.4 (Ret. 10). 


$$
\text { resolving power }=w / d w=8 \mathrm{~F}^{2} / \mathrm{h}^{2}
$$

$F$ is the focal length of the paraboloid collimator $(10.5 \mathrm{~cm})$ and $\mathrm{h}$ is the diameter of the source. The off-axis paraboloid reflector is located $12 \mathrm{~cm}$ from the glowbar and it is $7.5 \mathrm{~cm}$ in diameter.

The substrate for the beamsplitter is made of two matching potassisium bromide $(\mathrm{KBr})$ plates. $\mathrm{KBr}$ is a dielectric salt with index of refraction equal to 1.6. The actual beamsplitter is a thin layer of germanium film deposited on one of the $\mathrm{KBr}$ plates. This beamsplitter also acts as a low pass filter which allows radiation with energy less than the room temperature Ge band-gap (660meV or $5333 \mathrm{~cm}^{-1}$ ) to pass through. The $\mathrm{KBr}$ substrate acts as a high pass filter which allows radiation with energy more than $50 \mathrm{moV}\left(400 \mathrm{~cm}^{-1}\right)$ to pass through. Together they form a pass band of $400-5.333 \mathrm{~cm}^{-1}$. Behind the beamsplitter is the moving mirror assembly. This mirror is contrelled by a linear motor which scans at constant velocity. The reason for maintaining a constant velocity is to convert the spatial interferogram into a temporal one as illustrated by equation 2.5. This temporal inteferogram can then be processed by the electronics.

$$
[I(t)-0.5 I(0)]=0.5 I(0) \cos (4 \pi v t w)
$$

When a comparison between equation 2.5 and equation 2.1 is made, one sees that $l(x)$ is replaced by $l(t)$ and " $x$ " is replaced by "2vt" because the optical path difference is twice the physical path difference. During a scan, the detectcr samples data at fixed space intervals set by the reference interferometer. The set-up of the reference interferometer is similar to the main one, ercept that its source is a monochromatic HeNe laser. The interferogram of this single frequency source is a pure cosine with a spatial period equal to 6328 


\section{FTS-20E TOP VIEW}

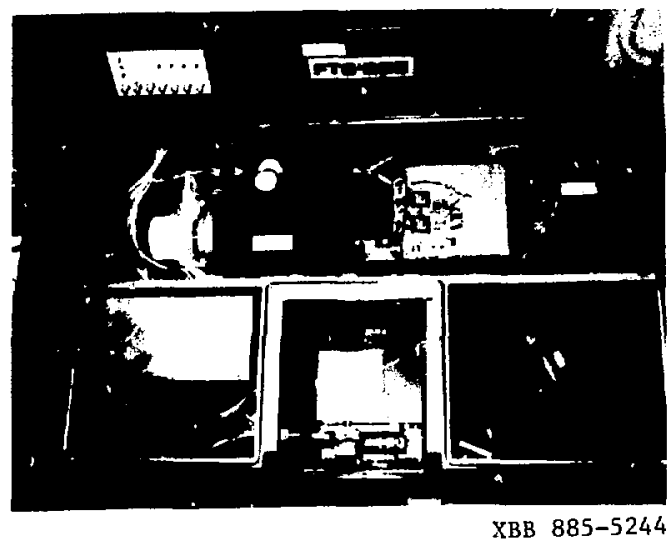

FTS-20E SCHEMATIC TCP VIEW

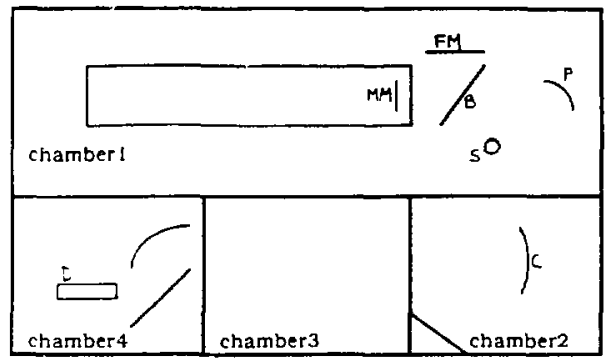

S--source

P--paraboloid mirror

B--beamsplitter

FM--fixed mirror

MM--meving mirror

C--converging mirror

D--detector

Fig. 2.3

Schematic of Digilab FTS-20E; chamber 1 contains the referince, the white light , and the main interferometer; chamber 2 contains two mirrors; chamber 3 is the sample chamber; and chamber 4 contains the built-in TGS detector system. 
angstroms. Synchronization between the main and the reference interferometer is achieved by utilizing the back of the main's moving mirror as the moving mirror of the reference. Zero crossing points of this cosine are used to trigger the collection electronics of the main interferometer. A discrete interferogram is collected at regular spatial intervals which equal to multiples of 6328 angstroms. A third interferometer, the white light interferometer is set-up in such a way that it shares the same fixed mirror, moving mirror and beamsplitter with the reference interferometer. The function of this white light interferometer is to insure that every interferogram recorded by the computer starts at the same point in real space. The zero path signal (maximum signal) recieved by the white light detector is at a fixed moving mirror position. As the moving mirror scarıs into this position, the white !ight detector enables the triggering circuits of the reference interferometer. Thus, the computer always records this point as the first data point. Successive scans of the sarie interferogram can be recorded coherently because they all start at the same point in real space.

Under normal operation conditions, the computer samples two tirises for each laser wavelength, which leads to an upper limit in frequency response of $7900 \mathrm{~cm}^{-1}$. Sampling at 6328 angstroms or higher multiples leads to aliasing or folding of the spectrum (for a detail discussion on aliased spectrum, see chapter 7 of reference 10). The length of the translation stage of the lirear motor is $6 \mathrm{~cm}$, which corresponds to a maximum optical delay of $12 \mathrm{~cm}$. The upper limit of resolutic n set by finite optical delay(d) is approximately $0.08 \mathrm{~cm}^{-1}$ (Ref. 10) as described by equation 2.6 .

resolution $=1 / d$

d stands for optical delay between the two beams. 
white light
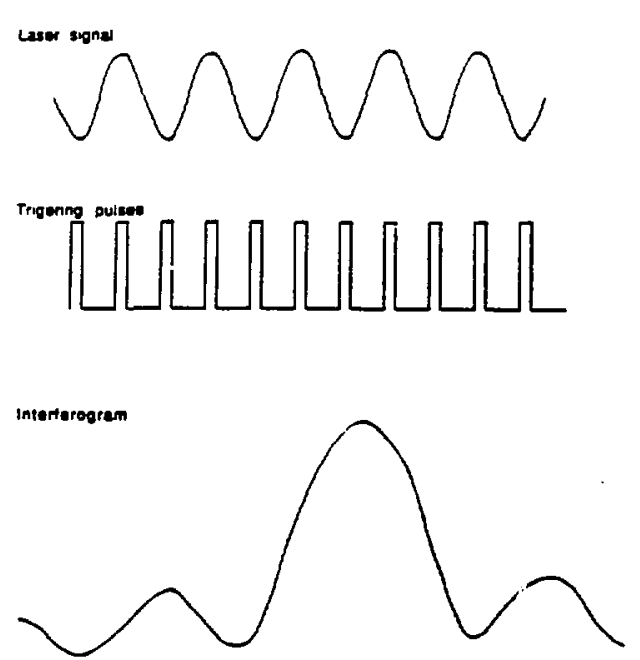

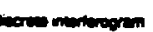

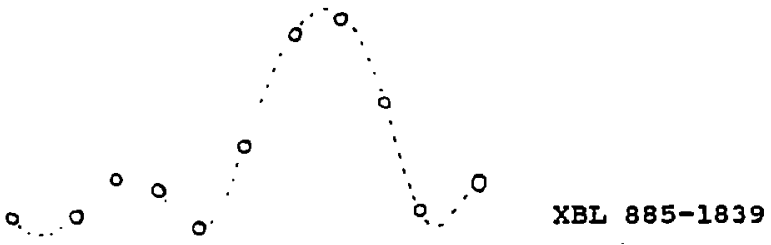

Fig. $2.4 \quad$ Zero crossings of lasers interferogram trigger the collection electronics to produce rectangular pusles, which enable the computer to record a discrete interferogram; data points located belore the white light's zero path is not recorded because the signal from the white light's zero path enables the triggering electronics. 
The converging mirror of the recombined beam is located at chamber two. It is $7.5 \mathrm{~cm}$ in diameter and has a focal length of $30.5 \mathrm{~cm}$. It focuses the recombined beam at the center of chamber three (the sample chamber) with a linear magnification factor of approximately three. Inside chamber four is the interferometer's built-in detector system. This system consists of an ellipsoidal mirror which images the focus inside the sample chamber onto the built-in detector (TGS) with a linear magnification factor of 0.1. Optical signals received by this detector will be converted into electrical signal, digitized by the electronics, and stored in the computer.

\section{3}

\section{AIR PRODUCTS LT-3-110 CONTINUOUS FLOW CRYOSTAT}

The built-in detector system mientioned above is not adequately set up for doing spatially resolved LVM spectroscopy of carbon in GaAs for two reasons. First, the finite size of the glowbar together with the focal length of the collimator reflector $P$ put a fundamental limit to the resolving power $(d w / w)$ of the interferometer to approximately 3000 as described earlier. At $582 \mathrm{~cm}^{-1}$ (the location of carbon's LVM in GaAs), this limits the resolution to approximately $0.12 \mathrm{~cm}^{-1}$, which is less than the maximum allowed resolution $\left(0.08 \mathrm{~cm}^{-1}\right)$ set by finite optical delay (Ref.10).The reasons for the necessity of this maximum resolution will be explained in the next chapter. Second, the size of the image $(30 \mathrm{~mm} \times 10 \mathrm{~mm})$ is too big for spatially resolved measurements. Both of the above problems can be solved by mounting the sample inside a specially desigrred liquid helium cryostat which is positioned at the focus of the image in chamber three (Fig 2.5). The cryostat used is a continuous flow liquid helium cryostat (Air Products LT-3-110). It consists of two major components: a continuous flow transfer line and the cryostat. The transfer line is a thermally 
shielded triaxial tube. Liquid helium is transfered from a nressurized dewar through the center tube. In order to minimized heat loss by evaporation in the center tube, liquid helium also flows through the intermediate space to act as a

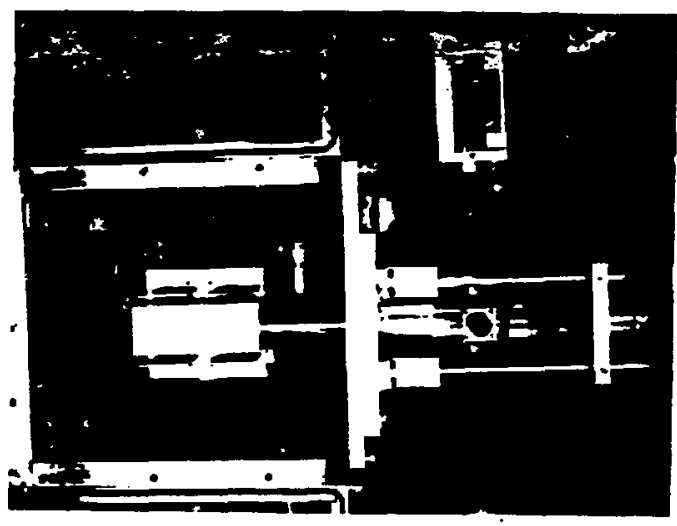

Fig. 2.5 Top view of cryostat moumted inside chamber 3 of the interferomeler (FTS-20E,.

heat shield. The outer tube space is usually evacuated to10E-5 torr or less to maximize insulation. Typical liquid helium consumption for this apparatus is approximately three liters per hour to maintain the temperature inside the cryostat near $4 \mathrm{~K}$. Figure 2.6 shows a cross sectional view of the cryostat. ' $O$ ' is the outer aluminium can. ' $B$ ' is a potassium bromide window which allows the recombined beam to enter the sample space without breaking the vaccum of the cryostat. ' $C$ ' is the intermediate shield coated with infrared biack paint to absorb any scattered radiation. ' $D$ ' is the cold finger maintained at $4 \mathrm{~K}$. The sample is mounted on the front side of the copper cold finger and is kept at $4 \mathrm{~K}$ so that prorion scattering of the sharp LVM absorption lines will not be 


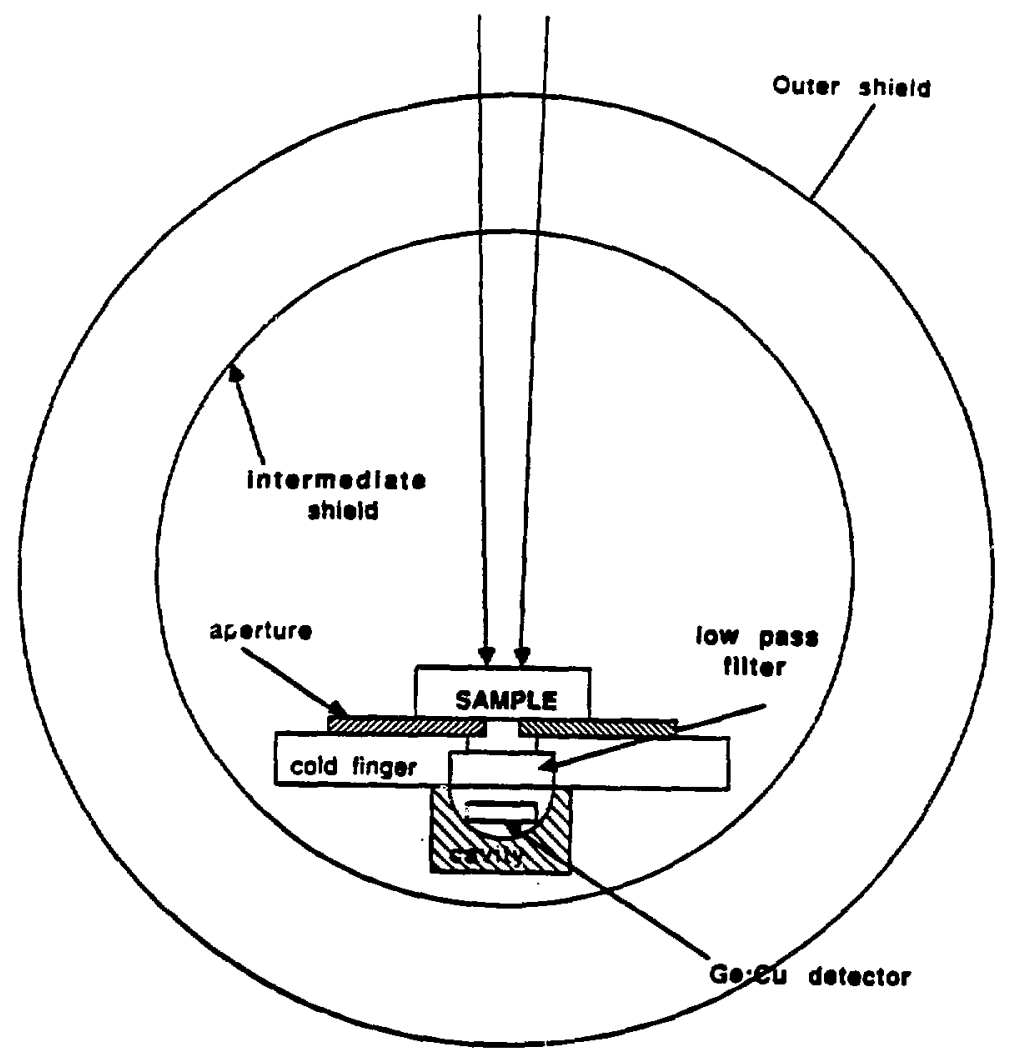

XBL 885-1840

Fig. 2.6

Cross sectional view of the continuous flow cryostat 
significant. In between the sample and the front surface of the cold finger is a copper plate $(0.2 \mathrm{~mm}$ thick) aperture (3mm in diameter) which serves two purposes. First, the aperture acts as a field stop for the detector so that it only sees $1 \mathrm{~mm}$ diameter of the source. This extends the finite source size restriction of resolving power to 30,000 , which translates into $0.02 \mathrm{~cm}^{-1}$ resolution at $582 \mathrm{~cm}^{-1}$. Therefore, the machine's resolution is now only limited by finite optical delay $\left(0.08 \mathrm{~cm}^{-1}\right)$. Second, it also acts as a field stop for the detector to see light coming from a frustum. The frustum's divergence angle matches the convergence angle of the focusing mirror $\mathrm{C}$. When the sample is mounted on the top of the copper aperture, spatially resolved measurments are made possible since only the region of the sample inside the frustum will be seen by the detector (Fig. 2.7). On the back surface of the cold finger is a multi-coated, germanium substrate low pass filter (OCLI-L13729-9) which lowers the upper band pass limit to $800 \mathrm{~cm}^{-1}$. This filter allows multiple "undersampling" or "aliasing" of the spectrum without introducing any erroneous features. Behind the filter is a brass cavity which houses a copper doped germanium photoconductive detector. Signal received by this detector is converted to a photocurrent, which in turn is amplified by a transimpedance amplifier (Fig. 2.8).

\subsection{OPERATION OF THE INTERFEROMETER PLUS CAYOSTAT FOR MEASURING SPATIALLY RESOLVED CARBON LVM}

The response of the photoconductor without the multi-coated low pass filter is shown in figure 2.9 by plotting intensity versus wavenumber. This spectrum has been collected at $4 \mathrm{~K}$ for ten minutes at $1 \mathrm{~cm}^{-1}$ resolution. Notice that $\mathrm{KBr}$ opening up at $400 \mathrm{~cm}^{-1}$. Figure 2.10 shows the same conditions but 


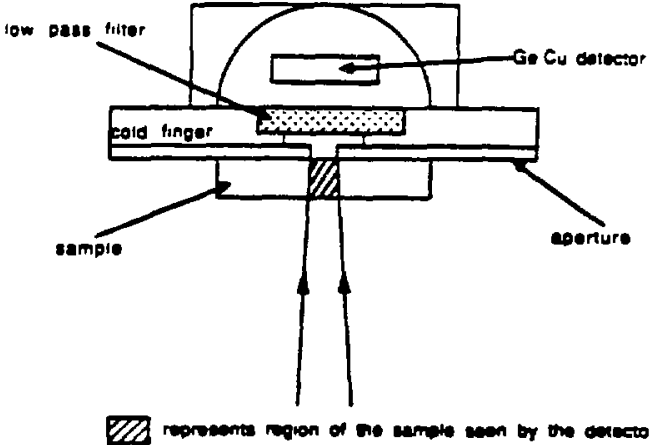

XBL $885-1841$

Fig. 2.7 Spatial mapping is achieved by using the copper plate aperture.

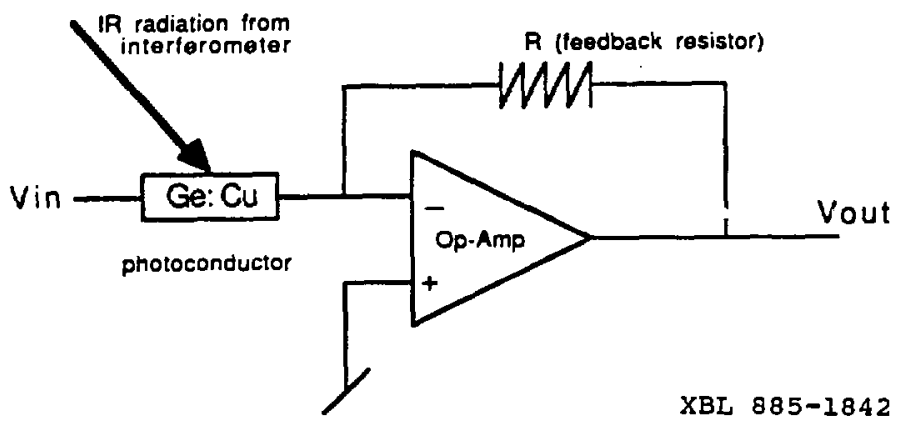

Fig. 2.8 Schematic of the transimpedance amplifier with the photoconductor. 
with the multi-coated low pass filter included. Only photons with frequencies between 400 to $800 \mathrm{~cm}^{-1}$ are reaching the detector. Figure 2.11 shows a transmission spectrum of GaAs. The broad absorption feature near $530 \mathrm{~cm}^{-1}$ is the characteristic 2 phonon absorption band (TO1+TO1,TO2+TO2, TO1+LO, TO2+LO) of GaAs (Ref. 13). Carbon LVM at $582 \mathrm{~cm}^{-1}$ can be seen as a single absorption line with $1 \mathrm{~cm}^{-1}$ resolution. With higher resolution, one observes that the carbon absorption line consists of five closely spaced components (the origin for 5 components will be discussed in the next chapter). The signal to noise ratio for this spectrum is approximately 20 for 2 hours of scanning time at $4 K$.

Because we want to determine the role of carbon as the shallowest compensating acceptor in semi-insulating GaAs, we want to know how carbon influences the electronic occupancy of EL2 as a function of position in SI GaAS wafers. That is , at any given location, we would like to know the total concentration of carbon. Furthermore, we would like to know what fraction of the carbon acceptors is ionized by shallow donors and what fraction is ionized by EL2. In order to achieve both objectives, we first "photo-quench" EL2 at temperatures near $4 K$. After quenching, the cryostat is positioned inside the interferometer with the sample at the position of the image of the source in chamber three to measure the charge state sensitive LVMs of carbon (the method we used to determine the charge state of the carbon acceptor from its LVM is discussed in chapter 3). The sample is then warmed up to room temperature and the next data point for the spatial map is collected by sliding the sample laterally along the radial direction with respect to the aperture. 


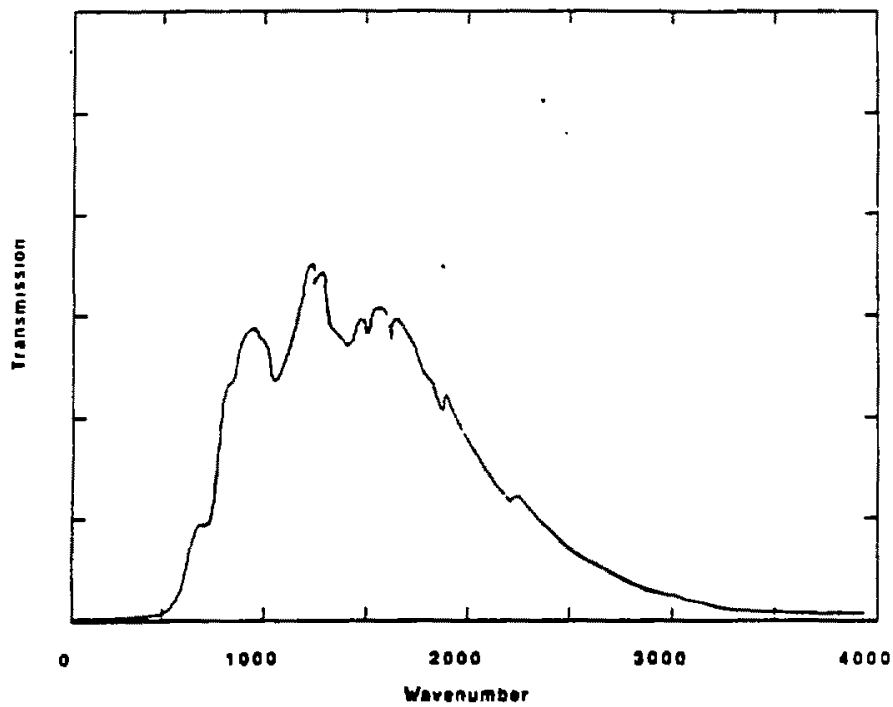

XBL $885-1843$

Fig. 2.9 Response of the copper.doped germanium photoconductor plolted as intensity versus wavenumber; experimental conditions are: resolution=1 $\mathrm{cm}^{-1}, 5 c a n n i n g$ time $=4 \mathrm{hr}$, temperature $=4 \mathrm{~K}$. 


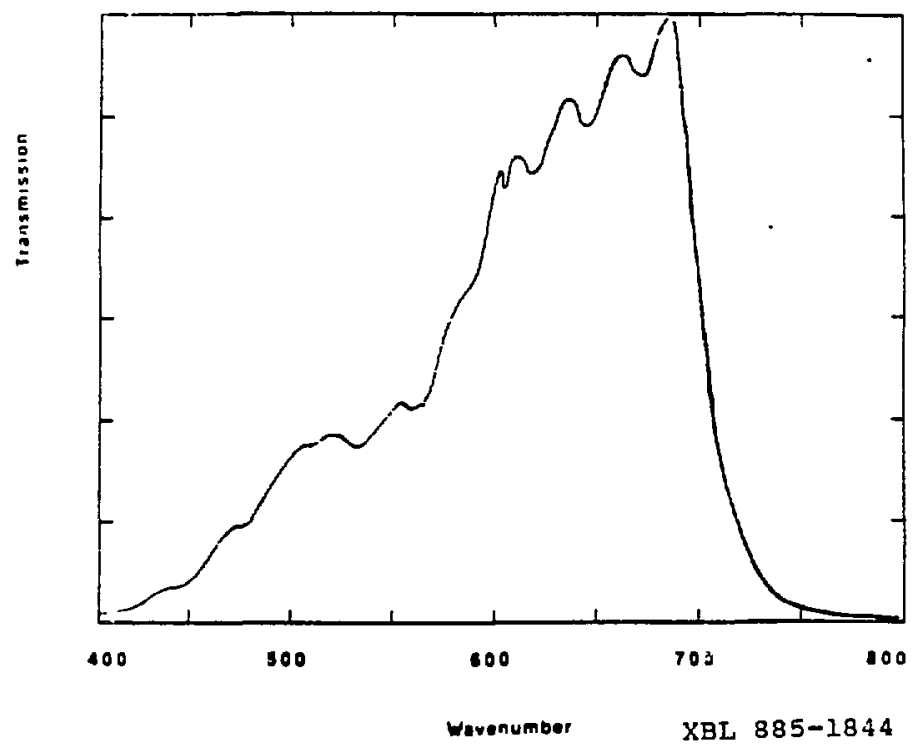

Fig. 2.10

Response of the copper doped Ge photoconductor with low pass optical filter; experimental conditions are identical as described in figure 2.9 . 


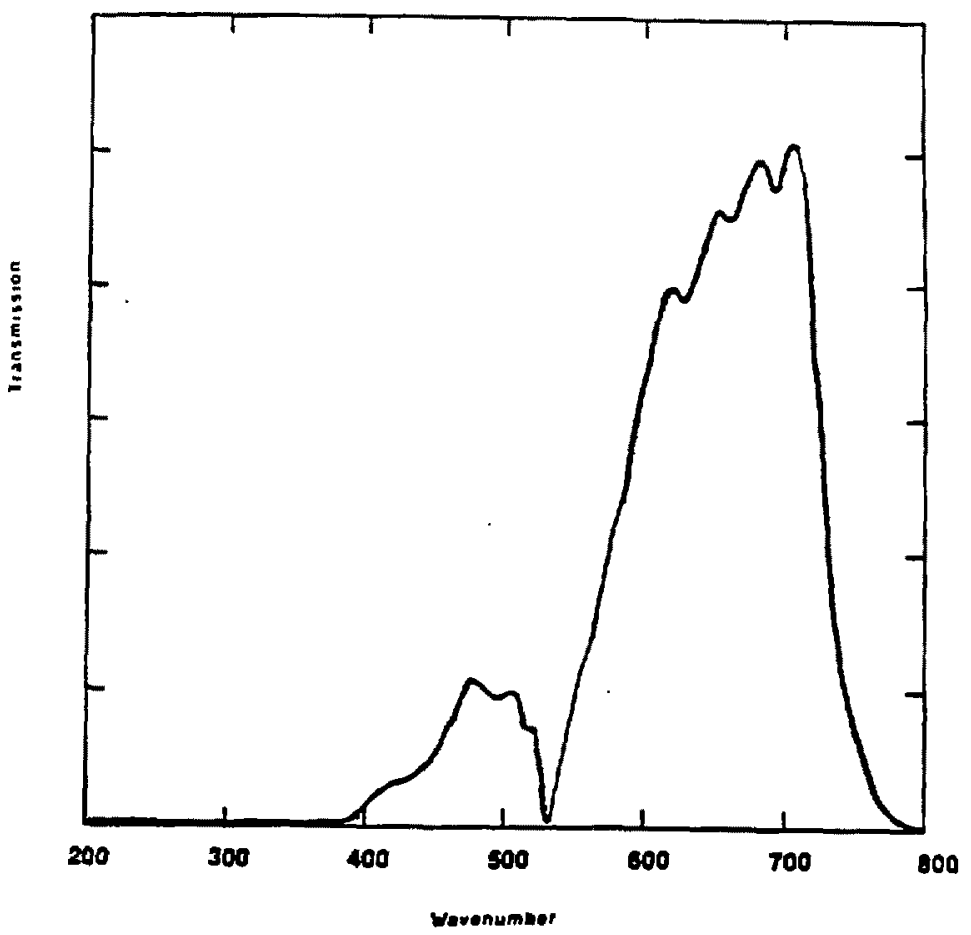

XBL $885-1845$

Fig. 2.11 Response of the photocondutor with low pass filter and a GaAs wafer; experimental conditions are identical as described in fig.2.9; important features are: band pass from 400 to $800 \mathrm{~cm}^{-1}$ and the 2 phonon band (see text) absorption near $530 \mathrm{~cm}^{-1}$. 
In this chapter, spatial maps of carbon's LVM in three as-grown semiinsulating LEC GaAs wafers will be presented. The samples include one twoinch diameter wafer $(0.5 \mathrm{~mm}$ thick) map and two three-inch diameter wafers ( $4 \mathrm{~mm}$ and $2 \mathrm{~mm}$ thick) maps. Before discussing these results, two background topics are given to familiarize the reader with the terms used to discuss these results. First, an introduction to linear absorption spectroscopy is given. Then, we will discuss how the $\mathrm{C}$ - and $\mathrm{C}^{0}$ fractions can be determined from LVM line shapes.

\subsection{LINEAR ABSORPTION SPECTROSCOPY}

All carbon absorption lines in GaAs preserited in this work are interpreted by the linear absorption law. In its simplest form, one can state that: (see fig. 3.1 for schematic)

$$
I(w)=I_{0}(w) \exp (-a(w) \times t)
$$

$I_{0}(w)$ stands for the flux of photons seen by the detector at frequency space inteval $w+d w$ if there were no defects present. $I(w)$ stands for the flux of photons seen by the detector at the same interval when defects are present in the sample and absorb some photons. ' $t$ ' is the thickness of the sample measured. The term ' $a(w)$ ' is known as the linear absorption coefficient at frequency $w$. It is directly proportional to the defect concentration when the absorption line of interest is fully resolved (eqn. 3.2):

$$
a(w)=c \times[\text { defects }]
$$



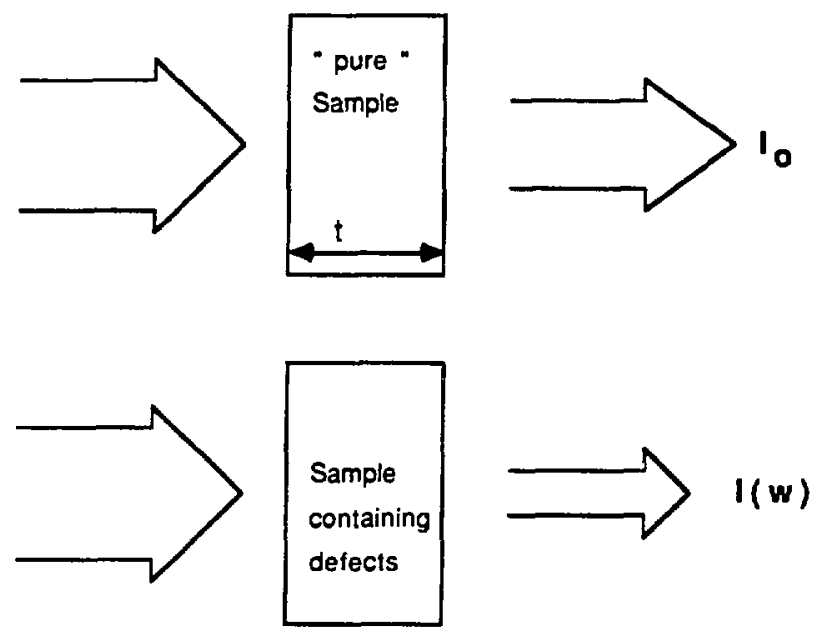

$$
I(w)=I_{0} \exp -(a t)
$$

XBL 885-1846

Fig. 3.1 Experimental contigurations for linear absorption spectroscopy: the top sample does not contain defects and a flux equal to to at frequency $w$ reaches the detector while the bottom sample contains defects and a smaller flux l(w) reaches the detector.

' $c$ ' is a proportionality factor known as capture cross section of the defect. If the absorption line of interest is underresolved, one needs to specify the resolution used to obtain ' $a(w)$ ' since it varies with resolution. If ' $a(w)$ ' is small (i.e. less than 0.1 ), one finds that ' $a(w) \times \Delta$ ' or the area under the absorption line $(A)$ in an absorption spectrum is independent of resolution used as demonstrated in figure 3.2. 

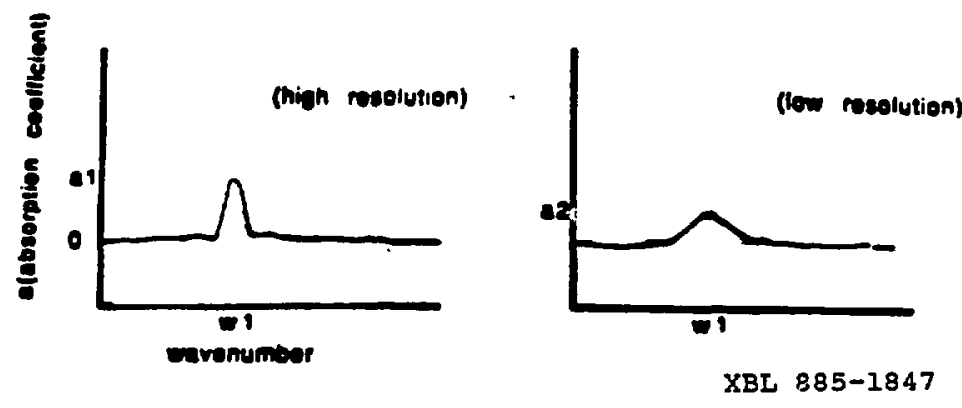

Fig. 3.2 Schematic showing that 'a $\times \Delta$ ' is preserved under different resolutions.

The term ' $\Delta$ ' denotes the full width at half maximum of the absorption line at a specific wavenumber resolution. Although other calibration values exist, the one used in this thesis is the value of $1.1 \times 10 \mathrm{E} 16 \mathrm{~cm}^{-1}$. (Ref.21)

$$
\text { [carbon] }=1.1 \times 10 \mathrm{E} 16 \mathrm{~cm}^{-1} \times A \text { or }(\mathrm{a} \times \Delta)
$$

( $A$ or $a x \Delta$ is in units of $\mathrm{cm}^{-2}$ )

The simple linear absorption law given by equation 3.1 is not exact since it neglects multiple internal reflections. Taking these reflections into account (with a plane parallel sample), one finds equation 3.4 to be a more exact description with 'r' standing for the reflectance at each sample vaccum interface.

$$
\begin{aligned}
& I=I_{0} \exp (-a t)\left\{\left(1-r^{2}\right) / 1-r^{2} \exp (-2 a t)\right\} \\
& r=[(n-1) /(n+1)]^{2} \text { at normal incidence }
\end{aligned}
$$


For calculations done in this thesis (with 'at' $\sim 0.1$ and ' $r$ ' $\sim 0.3$ ), equation 3.1 introduces less than $10 \%$ error. However, the calibration constant given in equation 3.3 is not more accurate than a factor of 2 , therefore one can safely assume that equation $\mathbf{3 . 1}$ gives acceptable results.

\subsubsection{Linear absorption spectroscopy of carbon's LVM in GaAs}

We have mentioned earlier that carbon LVMs c nsist of five closely spaced lines. The location of the lowest frequency line is at $58 ? . .4 \mathrm{~cm}^{-1}$ and the highest frequency line is at $583.05 \mathrm{~cm}^{-1}$ when measured at temperatures near $4 \mathrm{~K}$ with spectral resolution of $0.08 \mathrm{~cm}^{-1}$ or higher. It can be shown that nine different lines should exsist due to first neighbor interactions between carbon and the two naturaliy abundant isotopes of gailium $\mathrm{Ga}^{69}(60.4 \%)$ and $\mathrm{Ga}^{71}$ (39.6\%). There are three types of configuration for the two isotopes to arrange themselves around carbon.

(i) same isotope on all four neighbor sites: symmetry remains $T_{d}$, the triplet shifts, but does not split $\left(\Gamma_{5}, F_{2}\right)$ which results in 2 lines.

(ii) one isotope on the first neighbor site, the other one on the remaining three sites, the symmetry is lowered to $C_{3 v}$, triplet splits into singlet $\left(\Gamma_{1}, A_{1}\right)$ and doublet $\left(\Gamma_{3}, E\right)$ and results in $2 \times 2=$ 4 lines.

(iii) one isotope on two neighbor sites, the other on the other two sites, the symmetry is $C_{2 v}$ and there are 3 singlets $\left(\Gamma_{1}+\Gamma_{2}+\Gamma_{4}, A_{1}\right.$ $+B_{1}+B_{2}$ ) which results in 3 lines.

Howeve, only five distinct absorption lines can be observed since some of the 
lines superpose (Ref 14, 15). Figure 3.3 shows a transmission spectrum with a diode laser at $0.001 \mathrm{~cm}^{-1}$ resolution (Ref. 22). Even with this extremely high resolution, only five lines are observed.

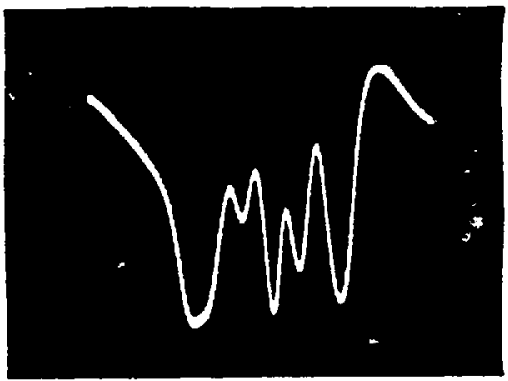

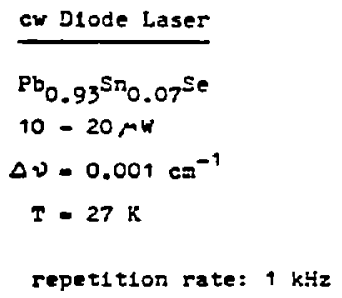

XBL $885-1848$

Fig. 3.3 Carbon's LVM as measured by a ciode laser at $0.001 \mathrm{~cm}^{-1}$ resolution at $27 \mathrm{~K}$ (Ret.22)

A typical spectrum obtained by Digilab FTS-20E is shown in figure 3.4 . It is a plot of transmitted intensity versus wavenumber. The experimental conditions for this particular run (and all data points in this thesis) are as follows:

(1) Cool sample to near $4 K$ inside the cryostat.

(2) Photo-quench EL2 away with tungsten lamp for 30 minutes.

(3) Measure carbon LVM ny Digilab FTS-20E for 400 co-added scans (2 hrs) with the sample r: intained near $4 \mathrm{~K}$.

(4) Warm up the sample.

(5) Slide the sample with respect to the aperture for the next data point. 


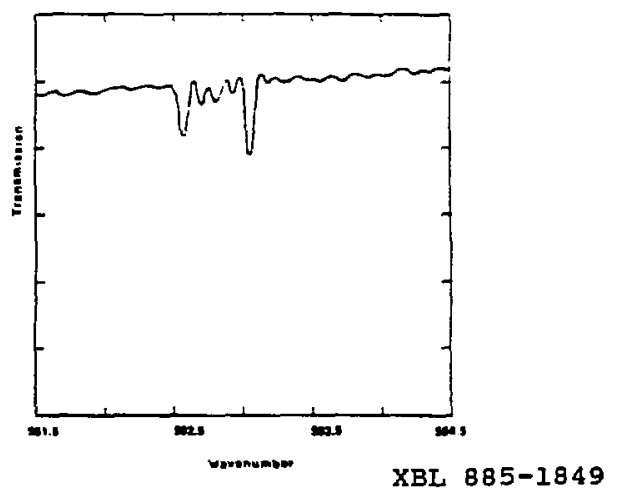

Fig. $3.4 \quad$ High resolution $\left(0.08 \mathrm{~cm}^{-1}\right.$ ) Iransmission spectrum of carbon LVM in GaAs; experimental conditions are given in the text.

This fully resolved spectrum was collected at $0.08 \mathrm{~cm}^{-1}$ resolution. In order to obtain an absorption spectrum (a plot of absorption coefficient versus wavenumber), one needs a carbon-free sample to obtain lo(w) mentioned in equation 3.1. Unfortunately, carbon free samples are not readily accessible and one needs to obtain $l o(w)$ by computer simulation. In figure 3.4, neglecting system noise, one finds that the local background intensity from $581 \mathrm{~cm}^{-1}$ to $585 \mathrm{~cm}^{-1}$ is relatively constant. Therefore, one can use this background intensity as $I_{0}(w)$ for computing the absorption spectrum (fig. 3.5). ' $a(w) \times \Delta$ ' for this set of absorption lines is defined by $A$ in equation 3.5 where $A$ stands for the area under the set of absoption lines:

$$
A=\Sigma A_{n} \Delta w \quad N \Delta w=583.1 \mathrm{~cm}^{-1}-582.4 \mathrm{~cm}^{-1}
$$

$A_{n}$ stands for the absorption value of the subinterval $\Delta w$ and $N$ stands for 
the number of subintervals (typically 10). The value $A$ for the sample shown in figure 3.5 is $0.1 \mathrm{~cm}^{-2}$ which corresponds to carbon concentration of $1.1 \times 10 \mathrm{E} 15$ $\mathrm{cm}^{-3}$. Detection limit for our experimental conditions is $5 \times 10 \mathrm{E} 13 \mathrm{~cm}^{-3}$ which is calculated by using a signal to noise ratio of 20 .

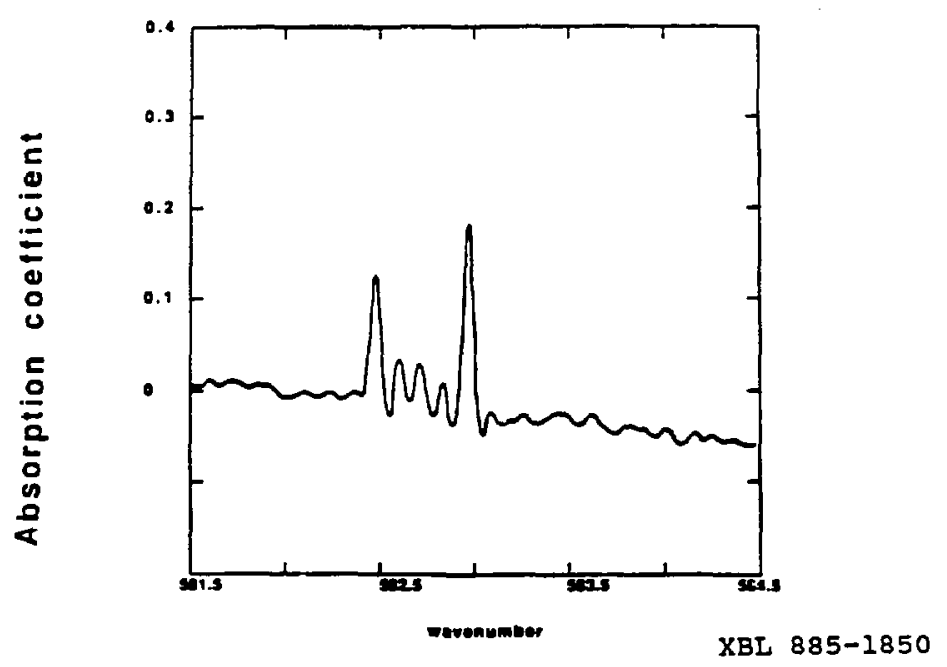

Fig. 3.5 Absorption spectum of fig. 3.4; background intensity is used as lo(w) to obtain this spectrum.

3.2

CHARGE STATE SENSITIVE LVM LINES OF CARBON IN GAAS

Besides concentration, fully resolved carbon LVM provides information regarding its charge state. Recent studies (Ref. 9) have shown that the LVM line of carbon at $0.06 \mathrm{~cm}^{-1}$ resolution may be decomposed into two independent The lines of the neutral state are significantly broader due to coupling between the bound hole elsctronic transition to the valence band and the LVMs. In 


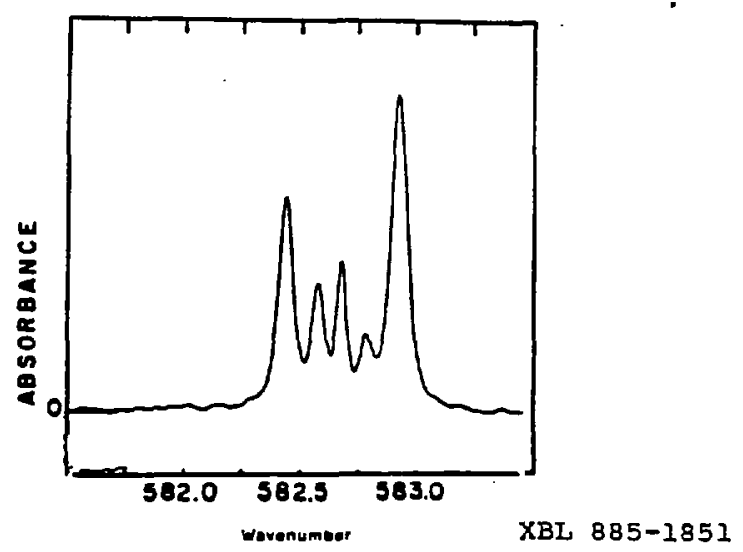

Fig. 3.6 LVM spectrum of ionized carbon acceptor in GaAs at $0.06 \mathrm{~cm}^{-1}$ resolution; important features are: 5 resolved lines with the lowest frequency peak at $582.4 \mathrm{~cm}^{-1}$.(Ret.9)

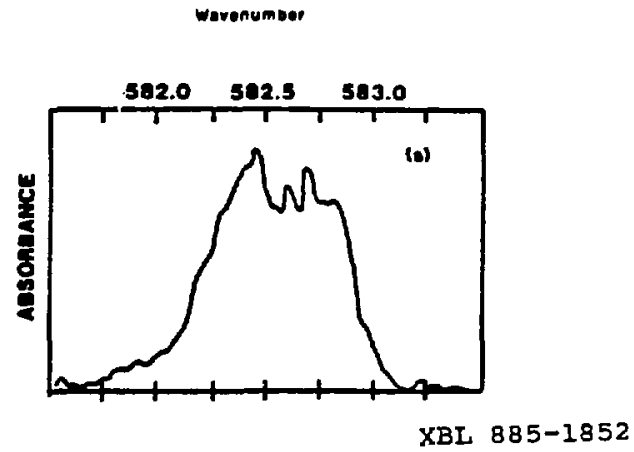

Fig. 3.7 LVM spectrum of neutral carton acceptors in GaAs measured at a resolution of $0.06 \mathrm{~cm}^{-1}$; all 5 peaks boarden and shilt approximately $0.15 \mathrm{~cm}^{-1}$ toward bwer frequencies (Ref. 9). 
addition, the bound hole also weakens the bonding of the carbon atom to the lattice. This effect results in a negative shift of $0.15 \mathrm{~cm}^{-1}$ in vibrational frequency. Any set of carbon LVM absorption lines is a linear combination of the neutral and the ionized component. Figure 3.8 shows a spectrum which consists of $80 \%$ reutral carbon LVMs and $20 \%$ negatively charged carbon LVMS.

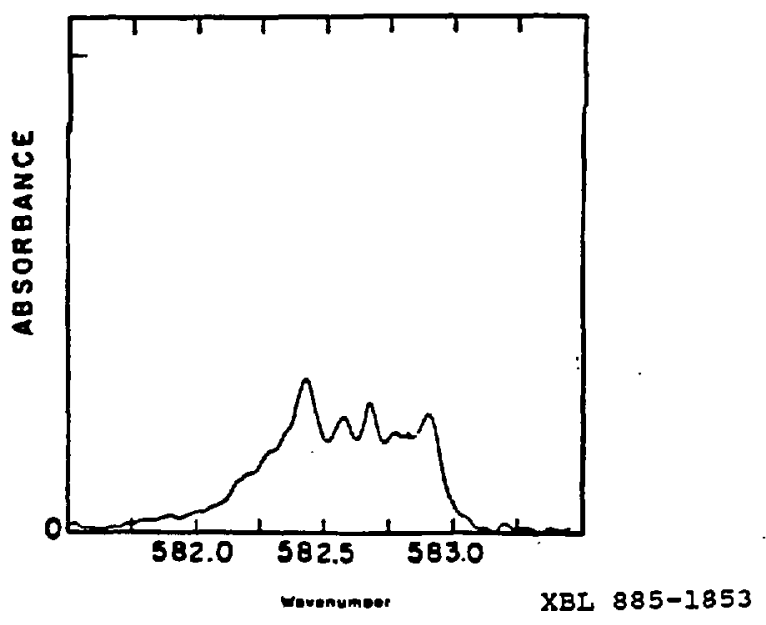

Fig. 3.8 Carbon acceptor LVM, $80 \%$ of the acceptors are neutral and $20 \%$ of them are ionized(Ref. 9)

The method used to decompose these spectrum into two components is discussed in reference 9. In this thesis, a phenomenological relation (eqn.3.6 (Ref. 16)) is used to determine the ratio of neutral carbon concentration to total carbon concentration. This relation is based on the fact that the ratio of the area under the absorption line of the lowest frequency component $\left(582.4 \mathrm{~cm}^{-1}\right)$ to that of the highest frequency component $\left(583.05 \mathrm{~cm}^{-1}\right)$ of fully resolved carbon 
LVM is strongly related to the charge state of carbon. Calling this ratio $R$, one can easily derive the ratio of neutral carbon concentration $\left[\mathrm{C}^{0}\right]$ to the total carbon concentration [C] by equation 3.6. (Notice that $R$ can be determined only if all 5 components are resolved; therefore, we must use $0.08 \mathrm{~cm}^{-1}$ or better resolution.)

$$
\left[C^{0}\right]=[C]\{(R-0.67) /(0.85 R-0.35)\}
$$

Utilizing equation 3.3 and 3.5, the results are summarized into six figures.

Figure 3.9------ carbon concentration profile for the 2-inch wafer

$$
\text { (0.5mm thick) }
$$

Figure 3.10---- ratio $A$ along the 2-inch wafer

Figure 3.11---- carbon concentration profile for 3-inch wafer $(4 \mathrm{~mm}$ thick)

Figure 3.12---- ratio $R$ along 3-inch wafer ( $4 \mathrm{~mm}$ thick)

Figure 3.13---.- carbon concentration profile for 3 -inch wafer $(2 \mathrm{~mm}$ thick)

Figure 3.14----- ratio $\mathrm{A}$ along 3 -inch wafer ( $2 \mathrm{~mm}$ thick)

The concentration profiles in figures $3.9,3.11$ and 3.13 are calculated in two steps. First, the .rea under the absorption line $A$ is determined by equation 3.5 for each data point. Then, each $\boldsymbol{A}$ is multiplied by the calibration constant given by equation 3.3 to obtain the concentrations. The relationship between each $R$ profile and the corresponding shallow donor concentration profile is discussed in chapter 4. 


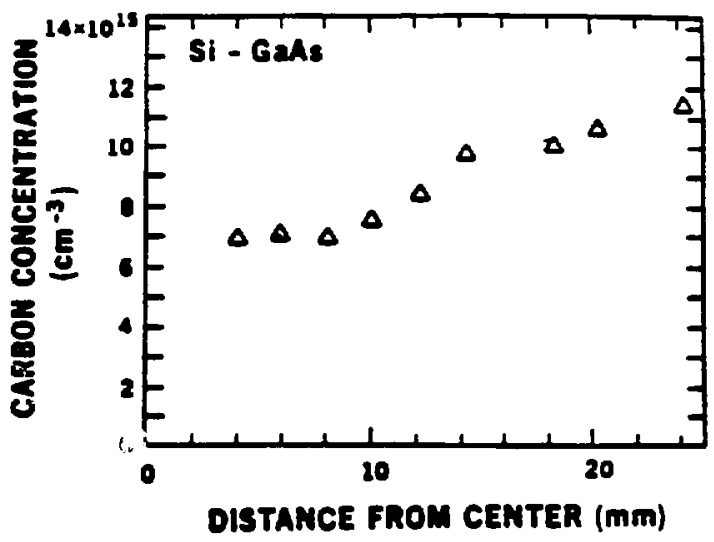

Fig. 3.9

Concentration profile of carbon in GaAs: maximum [carbon] (near the edge) is

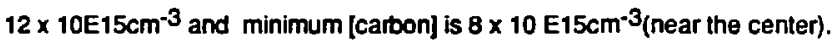

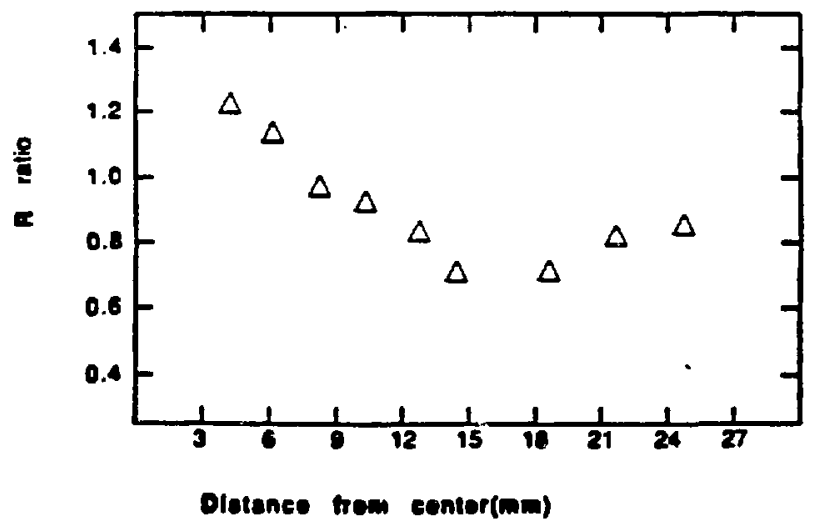

Fig. 3.10 Ratio $R$ along the radius of the water; it varies from 1.4 to 0.6 with maxima near the center and the edge and a minimum $15 \mathrm{~mm}$ from the center of the wafer. 


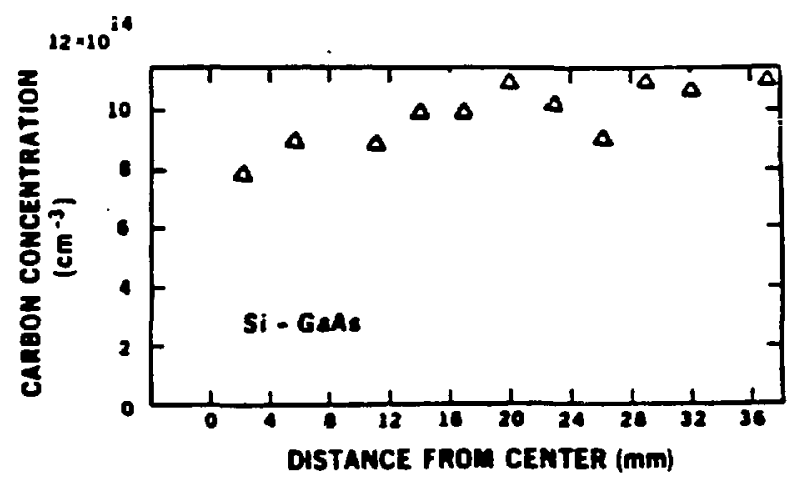

Fig. 3.11

Concentration profile of carbon along the radius of the $4 \mathrm{~mm}$ thick 3 inch diameter GaAs wafer; the profile is relatively coristant with an average [carbon] equal to $1 \times 10 E 15 \mathrm{~cm}^{-3}$.

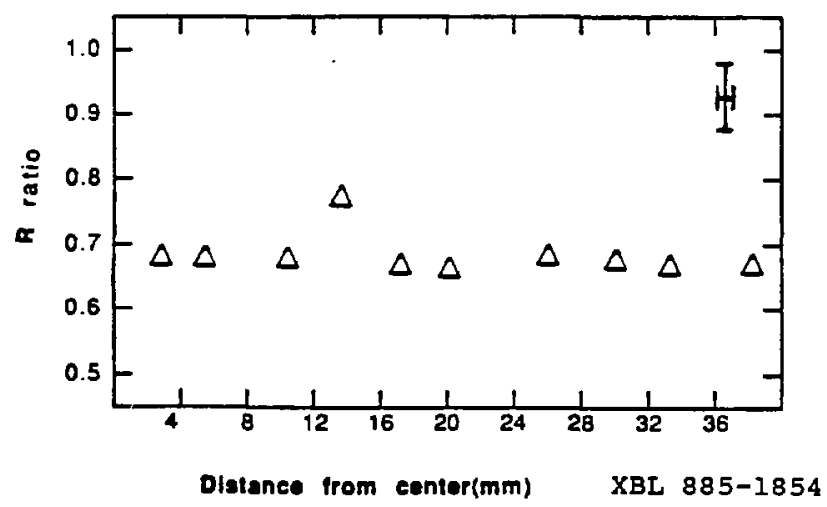

Fig. 3.12 Ratio $R$ along the radius of the $4 \mathrm{~mm}$ thick 3 inch diameter wafer; $R$ is relatively constant along the radius with an average value of 0.7 . 


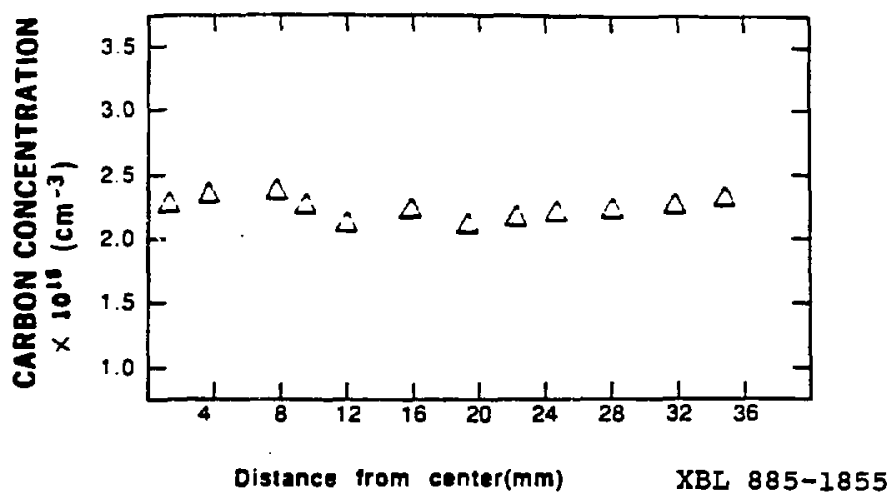

Fig. 3.13 Concentration profile of carbon abng the radius of the $2 \mathrm{~mm}$ thick 3 inch diameter GaAs water; the prolile is relatively constant with an average [carbon] of $2 \times 10 \mathrm{E} 15 \mathrm{~cm}^{-3}$.

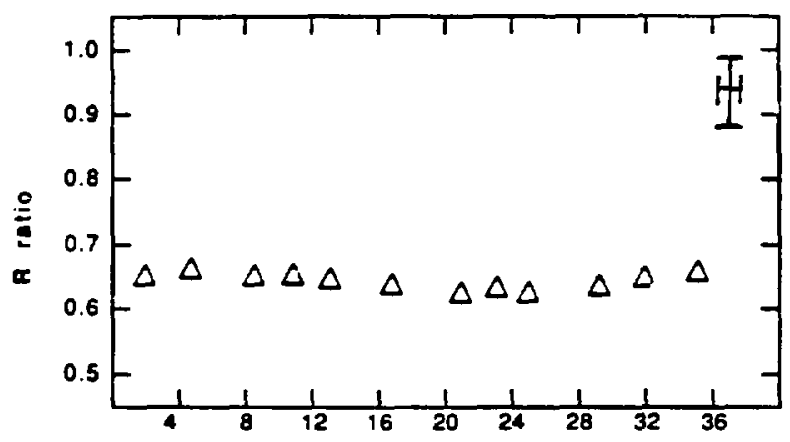

Distance from center(mm) XBL $885-1856$

Fig. 3.14 Ratio $R$ abng the radius of the $2 \mathrm{~mm}$ thick 3 inch diameter wafer; $R$ is relatively constam abng the radius with an average value of 0.67 . 


\section{DISCUSSION}

Because the electronic occupancy of EL2 depends on the balance between the concentrations of the major donors and acceptors, a detailed knowledge of the spatial profile of each major electrically active defect species is essential in understanding spatial the ' $W$ ' shaped variations of neutral EL2 concentration in GaAs. (Our discussion is based on the assumption of homogenous semiconductor crystal, i.e. we neglect band bending caused by dislocations.) However, one faces two major obstacies if one attempts to measure spatial profiles of each defect: first, many major defects: especially native defects, have not yet been characterized; second, the total number of distinct defect species is not always known. Therefore, one relies on inductive reasoning as an alternative to the deductive one: a hypothetical model is created first, then, spatial profiles of the known defects are tested against the model. If such model cannot incorporate the experimental results consistently, new models have to be created until the results can be incorporated without contradiction. In this chaptar, the same inductive reasoning is used to create models which incorporate the carbon spatial profiles presented in chapter three. In addition, spatial profiles of neutral EL2 at room temperature of the same wafers are measured to serve as a final test for consistency of these models. (Notice that all models must be consistent with the semi-insulating conditions (equation 4.1 and 4.2 )).

$$
\begin{aligned}
& \text { [acceptor } 1]+[\text { acceptor } 2]+\ldots . .>[\text { donor } 1]+[\text { donor } 2]+\ldots \ldots \\
& {[\text { [EL2] }+[\text { donor } 1]+[\text { donor } 2]+\ldots>[\text { acceptor } 1]+[\text { acceptor } 2]+\ldots .}
\end{aligned}
$$




\subsubsection{The "two defect" model for semi-insulating GaAs}

Since the total number of defect species inside the wafer is not known, the most logical model to start with is the simplest one--- the two defect model. In this model, one assumes that only one acceptor and EL2 exist in the wafer. The total concentration of EL2 is greater than the total concentration of carbon throughout the wafer as required by the semi-insulating criteria of equation 4.2 . Furthermore, one also assumes that both the carbon and the EL2 concentration are much greater than the concentration of all other electrically active defects.

For the two defects model, one assumes that two species with two charge states each, participate in the determination of the position of the Fermi level: neutral carbon acceptor $\left(\mathrm{C}^{0}\right)$, ionized carbon acceptor $\left(\mathrm{C}^{-}\right)$, neutral EL2 (EL20), and singly ionized EL2 (EL2+). At room temperature, all carbon acceptors should be ionized, the EL2 donors should be partially ionized and the Fermi level is pinned by EL2. The EL2 concentration profile in figure 4.1 is measured by room temperature $1.1 \mathrm{eV}$ near infraredabsorption of residual EL20 . It is important that one should not confuse such a profile with the total EL2 co.ıcentration profile. At temperatures near $4 \mathrm{~K}$ with EL2 "photo-quenched", most carbon acceptors should be neutral since the concentration of carbon is much larger than any residual donors as assumed by this model. However, experimental profiles of carbon in the two charge states near 4K with EL2 "photo-quenched" show that approximately $80 \%$ of the carbon acceptors are ionized near both the center of the wafer and the edge of the wafer (fig. 3.10). These experimental data clearly prove the existence of a major residual donor inside the wafer and we must abiandon the too simplistic two defect model. 


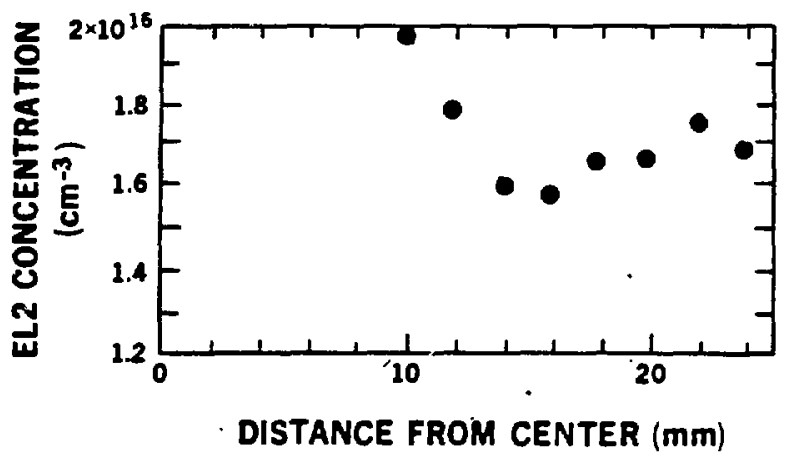

IBL $873-1065$

Fig. 4.1

Concentration profile of EL2 ${ }^{0}$ measured by $1.1 \mathrm{eV}$ photon absorption at room temperature; the profile has two local maxima (edge and center of the waler) and a minimum located al $16 \mathrm{~mm}$ from the center of the wafer; average concentration is $-1.7 \times 10 \mathrm{E}^{-16 \mathrm{~cm}^{-3}}$

\subsubsection{The "three defect model" for semi-insulating GaAs}

he next model considered is the "three defect model". Besides EL2, a donor shallower then EL2 is added. Three species with two charge state each participate in the positioning of the Fermi level. Besides $\mathrm{C}^{0}, \mathrm{C}^{-}, \mathrm{EL} 2^{\mathrm{O}}, \mathrm{EL2}+$, one has to take into account the new donor in its neutral state $D^{0}$, and its ionized state $D^{+}$. The semi-insulating criteria requires [carbon] > [donor] and [EL2] + [donor] > [carbon]. Under this condition carbon will be partially ionized at temperature near 4K with EL2 "photo-quenched". This is indeed the case shown in figure 3.10. With equation 3.6, one can calculate the spatial profile of the donors since the concentration of the donor at any location equals the 
concentration of ionized carbon when all EL2 are "photo-quenched". This donor profile is shown in figure 4.2. Important features of the donor profile include a minimum at $16 \mathrm{~mm}$ from the center of the wafer and two maxima located at the center of the wafer and at the edge of the wafer.

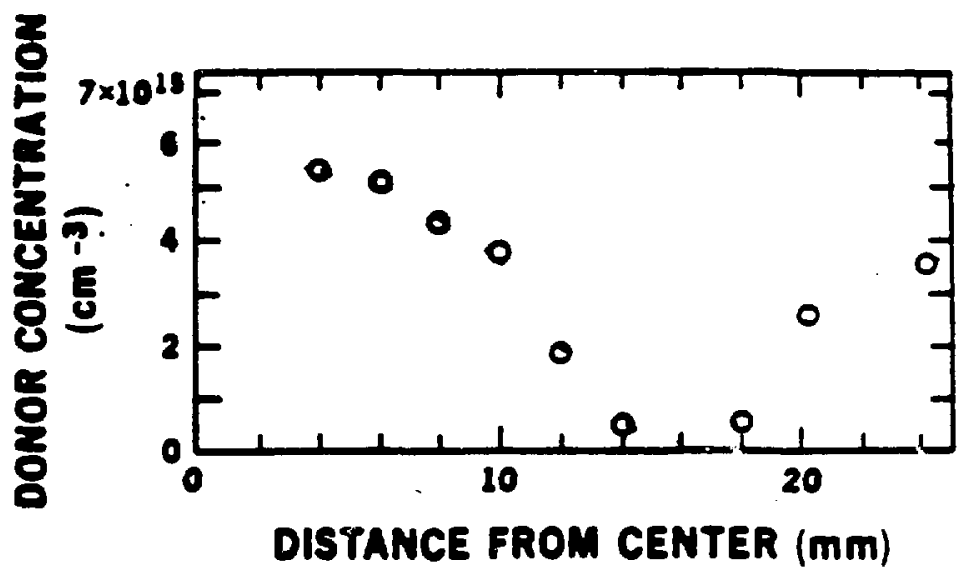

Fig. 4.2 Concentration profile of shallow residual donor predicted by the three delects model; this profile exihibits two local maxima and one minimum; the maxima are located at the center and the edge of the water while the minimum lies at 16 $\mathrm{mm}$ Irom the center.

Since the carbon concentration profile decreases slowly from the edge of the wafer to the center with a maximum to minimum ratio of two, the carbon profile contributes only weakly to spatial variations of neutral EL2 concentration. The donors, on the other hand, produce a greater effect because its maximum to minimum concentration ratio is bigger than 10 . If one assumgs the total EL2 profile to be relatively constant along the radius of the wafer, one expects the 
spatial profile of neutral EL2 to vary in accordance to the spatial profile of the donor only..That is, with a minimum located at $16 \mathrm{~mm}$ from the center of the wafer and two maxima (one at the edge and the other at the center) along the radius of the wafer. The overall shape of the neutral EL2 profile along the diameter of the wafer will be a " $W$ " shape with minima located at $16 \mathrm{~mm}$ from the center as reported by other authors (Fig.4.3) (Ref. 18). In order to verify this assumpiion, spatial profile of neutral EL2 at room temperature is measured. The result of this profile is already shown in figure 4.1. It is clear that the minimum of the profile is located at $16 \mathrm{~mm}$ from the center of the wafer and the assumption of a relatively constant total EL2 concentration is verified.

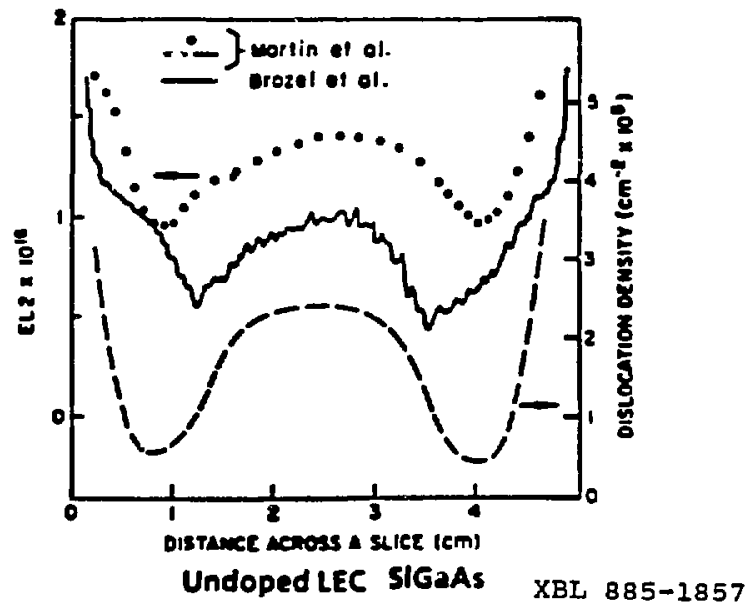

Fig. 4.3 Concentration protiles of neutral EL2 and dislocations across the diameter of various 2 inch GaAs waters (Ref. 18, Ret. 7).

\subsection{THE 4MM THICK THREE INCH DIAMETER WAFER}

4.2.1 The "two defect model" for semi-insulating GaAs

The first model considered is the two defect model. Once again, two 
species each with two charge state participate in the determination of the position of the Fermi level: $\mathrm{EL}^{0}, \mathrm{EL2}+, \mathrm{CO}^{0}$, and $\mathrm{C}^{-}$. The semi-insulating condition for this model is that the total concentration of EL2 is greater than that of carbon. In addition, the concentration of any residual defects should be much smaller than either of these. At temperatures near 4K with EL2 "photoquenched", most of the carbon acceptors are expected to be neutral. In figure 3.12 , the charge state profile of carbon acceptors indicates that all carbon acceptors are ionized at temperatures near 4K with EL2 "photo-quenched". This implies the existence of another major donor and the two defect model is invalid.

\subsubsection{The "three defect model" for semi-insulating GaAs}

The three defect model assumes the presence of an extra donor besides EL2 and carbon. The semi-insulating condition requires [carbon] $>$ [donor] and [EL2] + [donor] > [carbon]. Hence, one expects some of the carbon acceptors to be neutral when EL2 is quenched. But figure 3.12 clearly indicates that all carbon acceptors are ionized with EL2 photo quenched. Therefore, the concentration of the extra donor is greater than carbon and the semi-insulating condition is no longer satisfied if one assumes that the three defect model is correct.

\subsubsection{The "four defect model" for semi-insulating GaAs}

In this model, one assumes the existence of two acceptors and two donors. The semi-insulating criteria for this model requires [carbon] + [acceptor] $>$ [donor] and [EL2] + [donor] $>$ [carbon] + [acceptor] . The results of figure 3.12 can be incorporated into this model without contradictions since the concentration of the residual donor can be greater than carbon as long as the sum of both acceptors is greater than that of the donor. Unfortunatelv, the identity of both the second donor and the second acceptor are not known and 
their concentration profiles are not available. Nevertheless, one knows that the concentration of the second donor must be greater than the carbon concentration of $1.1 \times 10 E 15 \mathrm{~cm}^{-3}$. At the present time, one cannot relate the carbon concentration profile to the neutral EL2 profile measured by $1.1 \mathrm{eV}$ near infrared absorption at room temperature (Fig.4.4).

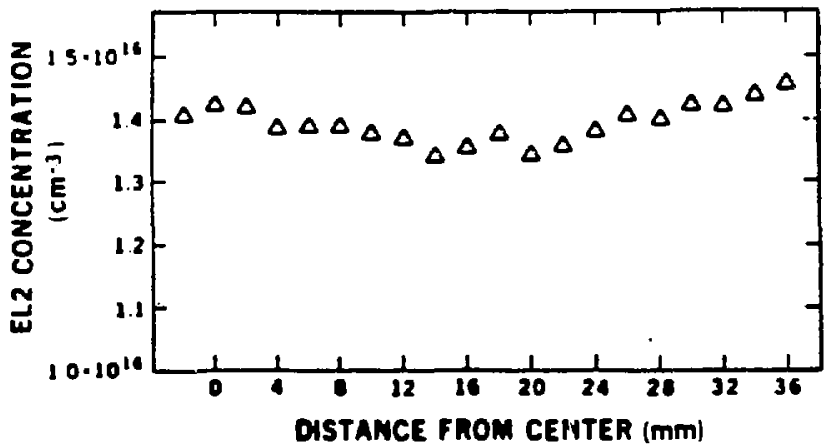

Fig. 4.4

Concentration profile of $E L 2^{0}$ measured by $1.1 \mathrm{eV}$ absorption at room temperature; the profile is relatively constant with an average value near $1.4 \times 10 \mathrm{E} 16 \mathrm{~cm}^{-3}$.

\subsection{TWO MM THICK THREE INCH WAFER \\ "Two" and "three defect models" for semi-insulating GaAs}

The carbon concentration and charge state profile obtained for this wafer is very similar to the $4 \mathrm{~mm}$ thick 3 inch wafer. The concentration profile for this wafer is a constant along the radius of the wafer and its average value is $2.2 \times$ 
$10 \mathrm{E} 15 \mathrm{~cm}^{-3}$. The charge state profile at temperatures near $4 \mathrm{~K}$ with EL2 "photoquenched" is also a constant, with the carbon acceptor fully ionized along the radius of the wafer. The charge state profile in figure 3.14 clearly rules out the two and three defect models as reasoned in section 4.2

\subsection{2 "Four defect model" for semi-insulating GaAs}

Because of the observed charge state profile(fig. 3.14), a four defect model is needed to incorporate this charge state profile consistently. As with the $4 \mathrm{~mm}$ thick 3 inch diameter wafer, the identity of the second donor and the second acceptor are not known and their concentration profiles are not available. The neutral EL2 spatial profile as measured by $1.1 \mathrm{eV}$ near infrared absorption at room temperature exhibits a " $W$ " shape across the diameter of the wafer (Fig. 4.5). However, one cannot relate the carbon concentration profile to this ' $W$ ' shaped variation without precise knowledge of the concentration profiles of the additional donor and acceptor.

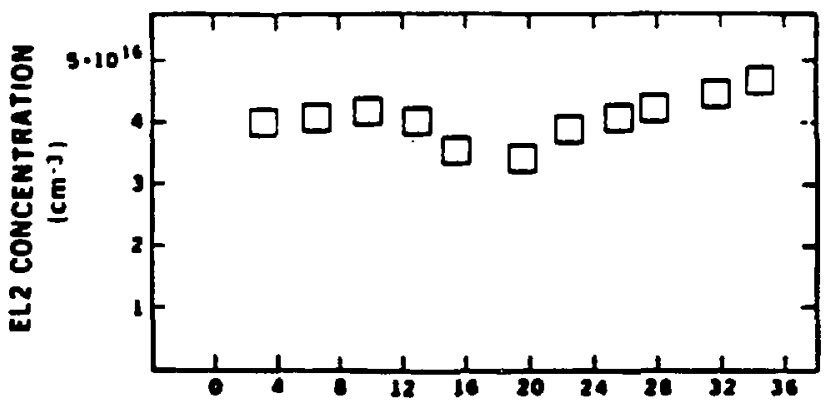

DSTANCE FROM CENTER (mm) XBL 885-1858

Fig. 4.5 Concentration profile of EL2 ${ }^{O}$ measured by $1.1 \mathrm{eV}$ absorption at room lemperature; the profile has two local maxima (edge and center of the wafer) and a minimum located at $16 \mathrm{~mm}$ from the center of the wafer; average concentration is near $4 \times 10 \mathrm{E} 16 \mathrm{~cm}^{-3}$. 


\section{CONCLUSICN}

Spatial profiles of three as-grown LEC GaAs wafers have been measured by localized vibrational mode absorption spectroscopy. For the two inch wafer, the carbon concentration is slightly decreasing from the edge of the wafer toward the center of the wafer with less than $50 \%$ variation. The average value of the carbon acceptor concentration is $8 \times 10 \mathrm{E} 15 \mathrm{~cm}-3$. The variation of neutral EL2 concentration along the diameter of the wafer measured by the 1.1 eV near infrared absorption at room temperature resembles a " $W$ " shape with the minima located at $16 \mathrm{~mm}$ from the center of the wafer. This variation is caused by an unidentified donor .

For both three inch diameter wafers, the carbon concentration profiles are approximately constant along the radius of the wafers. The average values of carbon concentration are $1.1 \times 10 \mathrm{E}_{15} \mathrm{~cm}^{-3}$ and $22 \times 10 \mathrm{E} 15 \mathrm{~cm}^{-3}$. The variation of both neutral EL2 profiles measured by $1.1 \mathrm{eV}$ near infrared absorption at room temperature is not understood since spatial profiles of the unidentified donor and acceptor are not available.

Because the carbon concentration profile of each of the three wafer is relatively constant (with maximum to minimum ratio less than two), the carbon acceptors are not very likely to cause large spatial variations of neutral EL2 concentration. Future efforts on improving the uniformity of electrical and optical properties of LEC semi-insulating GaAs wafers should be directed toward identifying and reducing the concentration of the additional donor and acceptor species. 


\section{Apponglix}

To derive equation 2.3, let the incident amplitude of the white light source on the beamsplitter be $E(X, W)$. For an infinitestimal spectral interval centered at an arbitrary wavenumber ' $w$ ', we can write down the equation A.1.

$$
E(X, w) d w=E_{0}(w) \exp (i(w t-2 p X w)) d w
$$

$E_{0}(w)$ is the magnitude of $E(X, w)$. In an interferometer after amplitude splitting, both beams travel distances $X_{1}$ and $X_{2}$. Each beam has undergone one reflection from and one transmission through the beamsplitter. Let ' $r$ ' be the reflection coefficient and ' $t$ ' be the transmission coefficient of the beamsplitter. The recombined amplitude is $E_{R}\left(X_{1}, X_{2}, w\right) d w$.

$$
\begin{aligned}
E_{R}\left(X_{1}, X_{2}, w\right) & =E\left(X_{1}, w\right) d w+E\left(X_{2}, w\right) d w \\
& =n E_{0}(w)\left[\exp \left(i\left(w t-2 p X_{1}\right)\right)-\exp \left(i\left(w t-2 p X_{2}\right)\right)\right] d w
\end{aligned}
$$

The flux for this particular infinitestimal spectral interval after recombination of the beams is $I\left(X_{1}, X_{2}, w\right) d w$.

$$
\begin{aligned}
I\left(X_{1}, X_{2}, w\right) d w & =Q\left[\left(E_{A}\left(X_{1}, X_{2}, w\right)\right)\left(E_{A}\left(X_{1}, X_{2}, w\right)\right)^{*}\right] \\
& =2 Q(r)^{2}\left(E_{0}(w)\right)^{2}\left\{1+\cos \left[2 \rho\left(X_{1}-X_{2}\right) w\right]\right\} d w
\end{aligned}
$$

$Q$ is a unit conversion constant. At any specific optical path difference $x=\left(X_{1}-\right.$ $\left.X_{2}\right)$, the total flux from all wavenumbers is $l_{1}(x)$. 


$$
\begin{aligned}
l_{1}(x) & =I\left(X_{1}, x_{2}, w\right) d w \\
& =2 Q(t)^{2} \int_{0}^{\infty}\left\{\left(E_{0}(w)\right)^{2} d w+\left(E_{0}(w)\right)^{2} \cos [2 p(x) w]\right\} d w
\end{aligned}
$$

Define the flux at $x=0$ as $I_{1}(0)$

$$
\left.I_{t}(x)-0.5 I_{t}(0)=\int_{0}^{\infty} 2 Q(t)\right)^{2}\left(E_{0}(w)\right)^{2} \cos [2 p(x) w] d w
$$

The spectrum of the source can be recovered by taking the Fourier cosine transform of equation A.5.

$$
\begin{aligned}
B(w) & =Q\left(E_{0}(w)\right)^{2} \\
& \left.=\int_{0}^{\infty}(\pi)^{2}(1 / p) l_{t}(x)-0.5 l_{t}(0) \cos [2 p(x) w]\right\} d x
\end{aligned}
$$

Neglecting the constant" $(t)^{2} n$, equation A.6 is the same as equation 2.3. 


\section{REFERENCE}

C. Kittel , "Intro. to Solid State Physics", 5th ed., John Wiley and Sons, New York(1976).

Landolt and Bornstein, "Numerical Data and Functional

Relationship in Scierice and Technology" Group III "Crystal and Solid State Physics" 17a, edited by 0 . Madelung, Springer-Verlag (1982).

D. E. Aspens, C. G. Olson, and D. W. Lynch, Phys. Rev. Letters 37 , 766 (1976).

A. M. White, P. J. Dean, D. J. Ashen, J. B. Mullin, M. Webb, B. Day, and P.D. Greene, J. Physics C E. L243 (1973).

5 R. C. Newman, F. Thompson, M. Hyliands, and R. F. Peart, Solid State Comm., 10, 505 (1972).

6 G. M. Martin, Appl. Phys. Letter 39747 , (1981).

7 M. R. Brozel, I. Grant, R. M. Ware, and D. Stirland, Appl. Phys. Letters $42,610,(1983)$.

8 W. M. Theis, K. K. Bajaj, C. W. Litton, and W. G. Spitzer, Appl. Phys. Letters 41,70 (1982).

9 B. V. Shanabrook, W. J. Moore, T. A. Kennedy, and P. P. Ruden, Phys. Rev. B 30,3563 (1984).

10 R. J. Bell, "Intr. to Fourier Transform Spectroscopy", Academic Press, New York(1972).

11 P. Jaquinot, Rep. Prog. Phys. 23, 267 (1960).

12 P. Felleget, J. Physics Radium 19, 181 (1958)

13 W. Cochran, S. J. Fray, F. A. Johnson, J. E. Quarrington, and N. Williams, J. of Applied Physics 32.2102 (1961). 
14 D. N. Talwar, M. Vandevyver, K. K. Bajaj, and W. M. Theis, Phys. Rev. B 33,85 (1986).

15 R. S. Leigh, and R. C. Newman, J. of Physics C 15, L1045 (1982).

16 W. Walukiewicz, E. Bourret, W. F. Yau, R. E. McMurray, Jr., E. E. Haller, and D. Bliss, Proc. "Defect Recognition and Image Processing in III-V compounds II(DRIP II)" April 27-9 1987, edited by E. Weber, Elsevier Science Publishing, New York(1987), p. 297.

17 W. Spitzer, "Advances in Solid State Physics", edited by O. Madelung, Pergamon, New York 1971.

18 G. M. Martin, G. Jacob, G. Poibland, A. Goltzene and A. Schwab, Inst. Phys. Ser 59, 2811 (1980)

19 M. Abe, T. Mimura, N. Yokoyama, and K. Suyama, Proc. "Solid State Devices 1982", Munich 13-16 Sept. 1982, edited by A. Goetzberger and M. Zerbst, Physik Verlag, Weinheim(1983), p.25.

20 J. B. Gunn, Solid State Comm. 1, 88(1963)

21 R. C. Newman, Proc. "European Materials Research Society" June 17-20,1986, Strasborg. France, in press.

22 K. H. Bachem, G. Knoll, J. Schneider, and H. Seelewind, Private Comm. 